\title{
Tropical Cyclone Climatology in a 10-km Global Atmospheric GCM: Toward Weather-Resolving Climate Modeling
}

\author{
Julia V. Manganello, ${ }^{*}$ Kevin I. Hodges, ${ }^{+}$James L. Kinter III, ${ }^{*},{ }^{*}$ Benjamin A. Cash, ${ }^{*}$

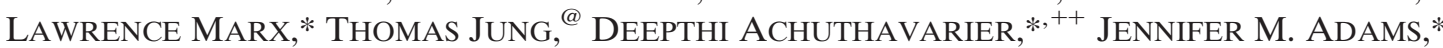

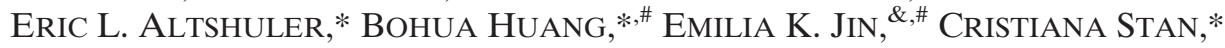 \\ PETER TOWERS, $* *$ AND NILS WEDI** \\ * Center for Ocean-Land-Atmosphere Studies, Calverton, Maryland \\ ${ }^{+}$National Centre for Earth Observation, NERC, University of Reading, Reading, United Kingdom \\ \# George Mason University, Fairfax, Virginia \\ @ Alfred Wegener Institute for Polar and Marine Research, Bremerhaven, Germany \\ ${ }^{\&}$ Korea Institute of Atmospheric Prediction Systems, Seoul, Korea \\ ** European Centre for Medium-Range Weather Forecasts, Reading, United Kingdom
}

(Manuscript received 15 June 2011, in final form 4 November 2011)

\begin{abstract}
Northern Hemisphere tropical cyclone (TC) activity is investigated in multiyear global climate simulations with the ECMWF Integrated Forecast System (IFS) at 10-km resolution forced by the observed records of sea surface temperature and sea ice. The results are compared to analogous simulations with the 16-, 39-, and 125-km versions of the model as well as observations.

In the North Atlantic, mean TC frequency in the 10-km model is comparable to the observed frequency, whereas it is too low in the other versions. While spatial distributions of the genesis and track densities improve systematically with increasing resolution, the $10-\mathrm{km}$ model displays qualitatively more realistic simulation of the track density in the western subtropical North Atlantic. In the North Pacific, the TC count tends to be too high in the west and too low in the east for all resolutions. These model errors appear to be associated with the errors in the large-scale environmental conditions that are fairly similar in this region for all model versions.

The largest benefits of the $10-\mathrm{km}$ simulation are the dramatically more accurate representation of the TC intensity distribution and the structure of the most intense storms. The model can generate a supertyphoon with a maximum surface wind speed of $68.4 \mathrm{~m} \mathrm{~s}^{-1}$. The life cycle of an intense TC comprises intensity fluctuations that occur in apparent connection with the variations of the eyewall/rainband structure. These findings suggest that a hydrostatic model with cumulus parameterization and of high enough resolution could be efficiently used to simulate the TC intensity response (and the associated structural changes) to future climate change.
\end{abstract}

\section{Introduction}

Tropical cyclones (TCs) - intense warm-core atmospheric vortices that form over the tropical waters and are accompanied by heavy rainfall and strong surface winds-belong to the category of extreme weather events.

${ }^{++}$Current affiliation: Universities Space Research Association, Columbia, Maryland.

Corresponding author address: Julia V. Manganello, Center for Ocean-Land-Atmosphere Studies, 4041 Powder Mill Road, Suite 302, Calverton, MD 20705.

E-mail: julia@cola.iges.org
Landfall of these systems can cause large human and material losses in many parts of the world. There has been a growing demand for climate models to accurately represent the statistical properties of TCs, as evidence suggests that these events may increase in intensity in connection with climate change (Solomon et al. 2007).

For decades, the ability of global atmospheric models to simulate TCs has been limited by, among other factors, inadequate horizontal resolution, which in turn has been limited by available computing resources. Although it has been known for some time that low-resolution models are capable of simulating hurricane-type vortices (see review by Walsh 2008), these systems differ substantially from observed TCs. Here "low resolution" 
refers to horizontal grids on the order of $100 \mathrm{~km}$ and larger, although the authors acknowledge that the exact definition is subject to debate and may change over time. Specifically, the intensity and the size of TCs depend crucially on the horizontal grid size with higher wind speeds and smaller scales at higher resolutions (e.g., Bengtsson et al. 2007b). The full three-dimensional structure of the storms also becomes more realistic as the resolution is increased.

An accurate representation of the TC intensity and structure requires grids that are fine enough to resolve sharp gradients in the inner core (eye and eyewall) of an intense storm where a typical eye diameter is on the order of $10-50 \mathrm{~km}$ (see Fierro et al. 2009 and the references therein). Numerical experiments with cutting-edge TC prediction models recommend grid spacing of less than $5 \mathrm{~km}$ (1-2 km preferred) (Chen et al. 2007; Fierro et al. 2009; Gentry and Lackmann 2010). Computing capabilities to run models at such high resolutions for a global domain over climate time scales of months to decades are currently virtually nonexistent. Other approaches, such as limited-area regional climate models (e.g., Knutson et al. 2007) and/or downscaling (e.g., Walsh 2008; Bender et al. 2010), have been employed in the meantime. While these approaches remain viable alternatives, global climate models are in principle a better tool to simulate TCs. This is because regional models are sensitive to several issues such as the choice of domain size, positioning of the boundaries, using only boundary conditions, or also enforcing the large-scale in the interior, to name a few, which could be the source of additional errors. These are not issues with global models. Most importantly, the twoway interaction between the TCs and the large-scale environment is preserved in the global modeling framework (see, e.g., Trenberth and Fasullo 2007).

In recent years, the finest-resolution global climate simulations have utilized grids in the range of 20 to $50 \mathrm{~km}$ (e.g., Oouchi et al. 2006; Zhao et al. 2009; Caron et al. 2010; Murakami and Sugi 2010) and have been performed with atmospheric general circulation models (AGCM) of relatively uniform resolution forced by sea surface temperature (SST) at the lower boundary. While some features of cyclogenesis and track density have been shown to improve in this resolution range (Caron et al. 2010), the common aspect of these simulations is that the most intense storms are not generated. In part, this could still be a consequence of insufficient resolution.

In this study, we use multiyear global AGCM simulations at multiple horizontal resolutions ranging from 125 to $10 \mathrm{~km}$ (see section 2) to investigate the benefits of fine resolution for simulating the characteristics of the Northern Hemisphere (NH) TC activity. The model used is the European Centre for Medium-Range Weather
Forecasts (ECMWF) Integrated Forecast System (IFS) operational weather forecast model, which is one of the most advanced models in terms of predictive skill and high spatial resolution (Bechtold et al. 2008). These very high-resolution global climate simulations have been performed as part of Project Athena (Kinter et al. 2011, manuscript submitted to Bull. Amer. Meteor. Soc.; Jung et al. 2012) in response to the call for a revolution in seamless weather and climate modeling made at the World Modeling Summit, held in May 2008 in Reading, United Kingdom (Shukla et al. 2009). This project brought together an international team of over 30 scientists to determine the feasibility of using dedicated highend computing resources to rapidly accelerate progress in simulating climate variability and global climate change.

The goal of this study is 1) to perform a systematic comparison of the TC statistics using a single AGCM integrated at widely varying and very high horizontal resolutions, extending thereby the resolution sensitivity studies of Bengtsson et al. (2007b), Caron et al. (2010), and Murakami and Sugi (2010) that investigated resolutions of up to T319, 0.3 degrees and T959, respectively; and 2) to determine whether there are any distinct improvements in the simulation of the TC intensity, structure, geographic distribution of cyclogenesis locations and tracks at the highest resolutions (10-16 km) in a model that still uses the hydrostatic approximation and parameterized cumulus convection (see section 2). Because of the computational constraints, ensemble simulations for the experiments analyzed have not been performed, which considerably limits the ability to study interannual TC variability (e.g., Vitart et al. 1997). For this reason, the focus of the present study is primarily on gross features of the TC climatology rather than variability, although some aspects of the TC variability are presented below.

This paper is organized as follows. Section 2 contains the description of the model and numerical experiments, including the model and observational data. Section 3 details methodologies of identifying and tracking TCs. In section 4, the climatology of TC formation and tracks for all available model resolutions is presented and discussed. The intensity distribution and the structure of tropical cyclones are described in sections 5 and 6, respectively. A short discussion of the interannual variability of the simulated TC statistics is included in section 7 , and a summary and conclusions are given in section 8 .

\section{Model, numerical experiments, and data}

The ECMWF IFS model consists of a dynamical component and a comprehensive physics package for processes requiring parameterization (European 
Centre for Medium-Range Weather Forecasts 2009). The dynamical component applies the spectral transform method to represent horizontal gradients with high accuracy while using a semi-implicit, semi-Lagrangian numerical scheme to step the equations forward in time (Temperton et al. 2001). In the vertical, a finite-element discretization using cubic B-splines as basis functions is employed (Untch and Hortal 2004). The physics package includes a cumulus convection parameterization that is a bulk mass flux scheme (Tiedtke 1989), which considers deep, shallow, and midlevel convection. Cloud and largescale precipitation processes are described by prognostic equations for cloud water/ice and cloud fraction and diagnostic relations for precipitation (Tiedtke 1993; Sundqvist 1978). Vertical turbulent transport is modeled using an eddy-diffusivity mass-flux approach (Köhler 2005). Land surface processes are represented by the revised land surface Hydrology Tiled ECMWF Scheme for Surface Exchanges over Land (HTESSEL) (European Centre for Medium-Range Weather Forecasts 2009).

Recent revisions to the physical parameterizations implemented in the IFS cycle Cy32r3, which is used in Project Athena, are described in Jung et al. (2010). They include the introduction of a convective entrainment rate proportional to the environmental relative humidity and the removal of the imposed strong coupling between the large-scale and the convection, and the variable convective adjustment time scale set proportional to the convective turnover time scale. These changes have led to higher levels of model activity with tropical variability becoming more realistic (see Bechtold et al. 2008).

For Project Athena, the IFS was integrated at four horizontal resolutions: spectral truncations of T159 (125 km), T511 (39 km), T1279 (16 km; currently used operationally to produce medium-range weather forecasts), and T2047 (10 km), each with 91 levels in the vertical. ${ }^{1}$ For the lowest three resolutions, 13-month hindcasts were carried out for 1960-2007, initialized on 1 November of each year. For the T2047 simulation, 13month hindcasts were performed for 1989-2007. No tuning or other changes to parameterizations were made between these model resolutions, except that in the model code, the convective adjustment time-scale has a resolution-dependent term. The SST and sea ice boundary conditions are the same $1.125^{\circ}$ data that were used for the 40-yr ECMWF Reanalysis (ERA-40) (Uppala et al. 2005). These data are monthly before 1990 and weekly starting in 1990 and are interpolated to daily values and in space to the IFS grid at each resolution. Beginning in 2002,

\footnotetext{
${ }^{1}$ Values given in parenthesis are the approximate spacings of the corresponding reduced Gaussian grids.
}

daily SST and sea ice from the ECMWF operational system are used. More details about the experimental design and overall climate simulation results are provided in Jung et al. (2012).

To keep data processing and storage at manageable levels, TC analysis is performed for the Northern Hemisphere (NH) and the part of the year from May to November (MJJASON season), which encompasses most of the TC season in the NH (e.g., Emanuel 2003). The analysis focuses on 19 years (1990-2008), which is the common period of all four experiments. For the lowest three resolutions, the analysis has also been extended to 30 years (1979-2008), and the results are presented where applicable.

To compare model results with global observations of TCs, we use data from the International Best Track Archive for Climate Stewardship (IBTrACS version v02r01, referred to hereinafter as IBTrACS; Knapp et al. 2010; available online at http:// www.ncdc.noaa.gov/oa/ ibtracs/). IBTrACS uses 10-min-average wind speed at 10-m elevation for the maximum sustained wind (MSW) estimate. This closely corresponds to the model definition of MSW (see section 3). We also use the same conversion coefficient between 1- and 10-min winds equal to 0.88 (see Knapp et al. 2010) to adjust TC thresholds. Thus, the "tropical storm" threshold of $17.5 \mathrm{~m} \mathrm{~s}^{-1}$ (34 kt) defined for the 1-min MSW becomes $15.4 \mathrm{~m} \mathrm{~s}^{-1}$ (30 kt) for the 10-min MSW. We also follow the definitions of the ocean basins used in the IBTrACS.

\section{Identification and tracking of tropical cyclones}

The initial TC identification and tracking is similar to that used in Bengtsson et al. (2007a) and is based on the tracking algorithm of Hodges (1995, 1999). It detects vortices in the $\mathrm{NH}$ as maxima in the $850-\mathrm{hPa}$ relative vorticity field (available every $6 \mathrm{~h}$ ) with values greater than $5 \times 10^{-6} \mathrm{~s}^{-1}$ (at a spectral resolution of T42). A post-tracking lifetime filter of 2 days is applied. The displacement distance of the selected vortices should satisfy a minimum of $10^{\circ}(\sim 1000 \mathrm{~km})$ over the lifetime of the vortex. This second filter primarily acts to remove monsoon depressions in the north Indian Ocean and extreme northwest Pacific. The IFS tends to produce an excessive number of these systems at all resolutions (not shown), which tend to be overly strong (see discussion in section $4 \mathrm{a}$ ) and therefore are identified as TCs without the displacement distance filtering. However, some slowmoving TCs in the Caribbean Sea and the Gulf of Mexico are also filtered out (not shown), the consequence of which is discussed later in section $4 \mathrm{~b}$.

To separate TCs from other synoptic systems, a number of criteria need to be satisfied. These generally 
TABLE 1. TC identification criteria.

\begin{tabular}{|c|c|c|c|c|}
\hline & \multicolumn{4}{|c|}{ Horizontal resolution of the IFS experiment } \\
\hline & T2047 & $\mathrm{T} 1279$ & T511 & T159 \\
\hline $\begin{array}{l}\text { 1. Surface }(10-\mathrm{m}) \text { wind speed threshold, } \mathrm{m} \mathrm{s}^{-1} \\
\text { (intensity threshold) }\end{array}$ & $15.4^{\mathrm{a}}$ & $15.4^{\mathrm{a}}$ & $14.1^{\mathrm{a}, \mathrm{b}}$ & $11.9^{\mathrm{b}, \mathrm{c}}$ \\
\hline $\begin{array}{l}\text { 2. Difference in vorticity between } 850 \mathrm{hPa} \text { and } 250 \mathrm{hPa} \\
\text { (a warm core condition) }\end{array}$ & \multicolumn{4}{|c|}{ Larger than zero for all resolutions } \\
\hline $\begin{array}{l}\text { 3. Vorticity max at each level between } 850 \text { and } 250 \mathrm{hPa} \\
\text { (a coherent vertical structure condition) }\end{array}$ & \multicolumn{4}{|c|}{ Applied to all resolutions } \\
\hline $\begin{array}{l}\text { 4. Criteria } 1-3 \text { are achieved for four consecutive } \\
\text { time steps }(24 \mathrm{~h})\end{array}$ & \multicolumn{4}{|c|}{ Applied to all resolutions } \\
\hline $\begin{array}{l}\text { 5. Cyclogenesis (first identification) occurs between } \\
0^{\circ}-20^{\circ} \mathrm{N} \text { over land and } 0^{\circ}-30^{\circ} \mathrm{N} \text { over oceans }\end{array}$ & \multicolumn{4}{|c|}{ Applied to all resolutions } \\
\hline \multicolumn{5}{|c|}{$\begin{array}{l}\text { a Observed "tropical storm" threshold for 10-min MSW is used. No correction for model time step is made for T2047 (7.5-min time step), } \\
\text { T1279 (10-min time step), and T511 (15-min time step). }\end{array}$} \\
\hline
\end{tabular}

include 1) a structural requirement for a storm to have a warm core to exclude midlatitude cyclones and other tropical systems that are not TCs and 2) an intensity threshold imposed on surface or low-level wind speed, or low-level vorticity, along with the formation region and duration requirements. Although there is no unique way to define TCs in a model simulation, in many studies the magnitudes of the chosen criteria are not well justified or explained (Walsh et al. 2007). We believe that there are essentially two ways to approach this issue: either the model TC identification criteria need to closely match the observational ones, and therefore the focus should be on the surface characteristics of TCs; or calibration of TC counts against observations (or reanalyses) needs to be performed to obtain the magnitude of preferred detection thresholds. We have chosen to focus on the first approach, as our goal is to directly assess model results against observations. In this case, our TC identification criteria generally follow the guidelines of Walsh et al. (2007) (see Table 1). Application of this set of criteria could also be viewed as a tough test of a model's ability to generate a sufficient number of intense TCs-a test that the model needs to pass to be considered for climate change simulations. To apply the conditions specified in Table 1, all initial tracks are referenced to the vorticity field at all available levels between 850 and $250 \mathrm{hPa}$ and at $\mathrm{N} 80$ resolution common to all experiments, using a steepest ascent maximization. ${ }^{2}$ This is performed recursively starting at $850 \mathrm{hPa}$ and using the

\footnotetext{
${ }^{2}$ Reference is made to the N80 full Gaussian grid, which has 320 longitudinal grid points (independent of the latitude) and 160 latitudinal grid points, symmetric about the equator.
}

maxima found at the previous level as a starting point. Steepest descent minimization is employed to reference tracks to the full resolution minimum sea level pressure (SLP), and a direct search is used to reference tracks to the full resolution maximum $10-\mathrm{m}$ wind speed. All referencing of the tracks to other fields is performed with a search radius constrained to $5^{\circ}$ of the storm center. For the direct comparison with observations, IBTrACS data are processed in a similar fashion imposing criteria 1 and 4 of Table 1.

\section{Climatology of tropical cyclone formation and tracks}

We begin our analysis of the model TC activity by comparing simulated and observed mean TC frequency along with the geographical distributions of cyclogenesis and track densities. The mean TC count, either for the $\mathrm{NH}$ as a whole or each basin examined, increases with the model resolution, in agreement with Caron et al. (2010). The total NH mean TC frequency is too low at T159, fairly realistic at T511 and too high at T1279 and T2047 for both periods of analysis (see Table 2). However, in the North Atlantic and the northeast Pacific, the model tends to produce a realistic number of TCs at the highest two resolutions, particularly at T2047. There are generally too few TCs in these basins at T511 and even fewer at T159, which is a known property of low-resolution global models (e.g., Camargo et al. 2005). The number of TCs is excessive in the northwest Pacific and the north Indian Ocean in all simulations except for T159. Although the relative contribution of the TC counts to the NH totals is consistently too high in the northwest Pacific and too low in the northeast Pacific and the North 
TABLE 2. Climatological mean and standard deviation (std) of the TC frequency per ocean basin for the MJJASON season during 19902008 and 1979-2008 (in parenthesis) for IBTrACS (Obs) and IFS simulations. Units are numbers per season. Differences between the model results and the corresponding observational values that are statistically significant at the $95 \%$ confidence level, using a two-sided Student's $t$ test, are shown in bold.

\begin{tabular}{|c|c|c|c|c|c|c|c|c|c|c|}
\hline & & & \multicolumn{8}{|c|}{ IFS } \\
\hline & \multicolumn{2}{|c|}{ Obs } & \multicolumn{2}{|c|}{$\mathrm{T} 2047$} & \multicolumn{2}{|c|}{ T1279 } & \multicolumn{2}{|c|}{ T511 } & \multicolumn{2}{|c|}{ T159 } \\
\hline & Mean & Std & Mean & Std & Mean & Std & Mean & Std & Mean & Std \\
\hline $\mathrm{NH}$ & $55.4(54.1)$ & $6.1(6.5)$ & 73.7 & 7.1 & $62.3(62.0)$ & $6.0(5.9)$ & $59.6(\mathbf{5 7 . 9})$ & $6.8(6.5)$ & $42.7(42.7)$ & $5.1(5.6)$ \\
\hline North Atlantic & $12.5(11.1)$ & $4.4(4.2)$ & 10.7 & 3.0 & $9.2(9.0)$ & $2.9(2.7)$ & $7.2(7.1)$ & $3.0(2.8)$ & $5.3(5.0)$ & $2.5(2.5)$ \\
\hline Northeast Pacific & $14.8(15.4)$ & $4.0(4.3)$ & 12.9 & 3.3 & $12.7(\mathbf{1 2 . 4})$ & $4.1(3.9)$ & $11.3(10.9)$ & $4.1(3.6)$ & $5.9(6.1)$ & $2.3(2.7)$ \\
\hline Northwest Pacific & $23.8(23.6)$ & $4.5(3.9)$ & 39.0 & 5.8 & $31.2(30.6)$ & $4.4(5.1)$ & $32.1(31.0)$ & $4.8(4.9)$ & $25.7(\mathbf{2 6 . 3})$ & $5.6(5.7)$ \\
\hline North Indian & $4.4(3.9)$ & $2.1(1.9)$ & 9.9 & 3.4 & $8.7(9.4)$ & $2.2(2.5)$ & $7.9(8.0)$ & $2.9(2.4)$ & $4.8(4.5)$ & $2.5(2.2)$ \\
\hline
\end{tabular}

Atlantic across all resolutions, similar to other models (e.g., Camargo et al. 2005; Bengtsson et al. 2007a; Zhao et al. 2009), this bias tends to decrease with higher model resolution (not shown).

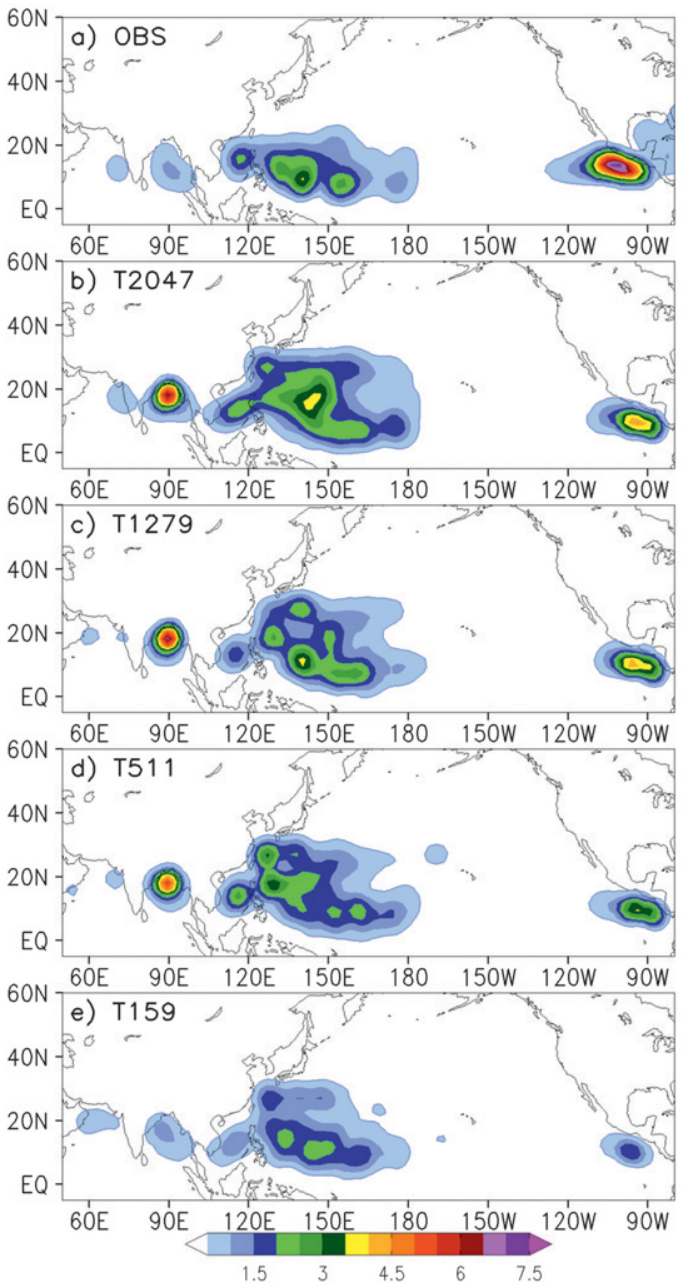

The geographic distribution of TCs is shown for genesis and track density for the North Pacific and the North Indian Ocean (Fig. 1) and for the North Atlantic (see Fig. 3), computed from the IBTrACS data and the

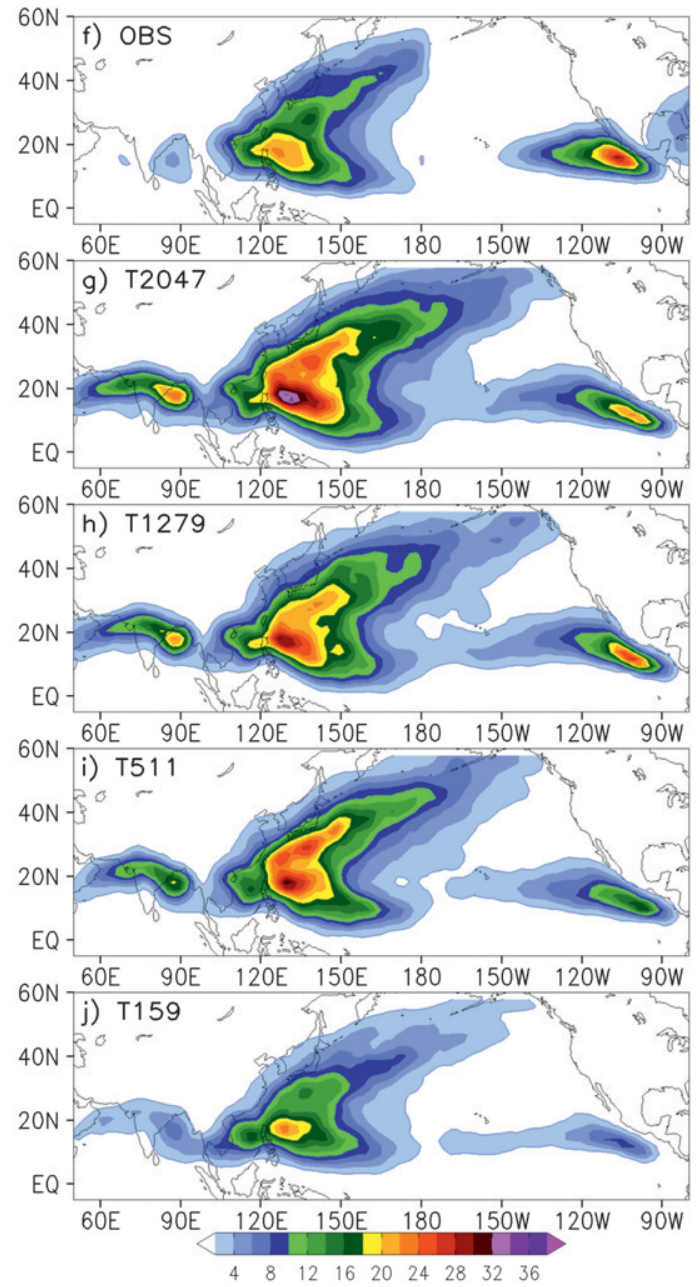

FIG. 1. North Pacific and the north Indian Ocean (left) genesis and (right) track densities as number density per season per unit area equivalent to a $5^{\circ}$ spherical cap for (a),(f) IBTrACS (Obs) and IFS simulations at (b),(g) T2047, (c),(h) T1279, (d),(i) T511, and (e),(j) T159 resolutions. 
generated cyclone tracks using the spherical kernel method (Hodges 1996). A direct comparison of the simulated distributions based on the original tracks with the observed ones, however, is difficult because of the differences in the cyclogenesis definition between the model and the observations. In the model, cyclogenesis is defined by the appearance of a weak initial vortex (see section 3) with a surface wind speed generally lower compared to the first records of the observed TCs (corresponding to the tropical depression stage of the development); also the observed TC tracks tend to be truncated once the identification criteria fail to be fulfilled even if the storm continues as an extratropical cyclone. Therefore, model storms contain both earlier and later stages of a life cycle than the observed storms. To reduce these differences, the genesis and track statistics shown in Figs. 1 and 3 were computed using model tracks modified to be more in line with the observations. That is, model cyclogenesis was redefined based on the surface wind speed magnitude that is most frequently found in the first records of the observed TCs. This value is equal to $11.3 \mathrm{~m} \mathrm{~s}^{-1}$ for the North Atlantic and the northeast Pacific and $6.8 \mathrm{~m} \mathrm{~s}^{-1}$ for the northwest Pacific and the north Indian Ocean on the basis of the IBTrACS data for 1990-2008. The first few records in the model TC tracks were therefore omitted if the simulated surface wind speed was below the corresponding threshold, specified above, adjusted for model resolution in the T511 and T159 cases. We must note that no adjustments to the decaying stages of the model TCs were made, that is, model cyclolysis was not redefined, which could be the source of some of the differences between the simulated and the observed track densities.

\section{a. North Pacific and the north Indian Ocean}

In the northwest Pacific, the genesis density is well simulated in all model integrations, although there are distinct deficiencies at the lowest two resolutions (Fig. 1, left panels): the region of the highest concentration of cyclogenesis is shifted more to the northwest at T511, and the center in the South China Sea is too weak at T159. The corresponding track density shows higher than observed concentration of tracks, except for T159 (Fig. 1, right panels), partly as a consequence of simulating too many TCs in this basin.

The northeast Pacific area of cyclogenesis is weaker than in the observations in all model integrations, particularly for the lowest resolution of T159 (Fig. 1, left panels, and Table 2), and is somewhat shifted to the southeast. Tracks display higher density in the central Pacific (Fig. 1, right panels), especially for T2047 and T1279. This is partly related to more active cyclogenesis in the northeast Pacific at the highest two resolutions, in addition to model storms being tracked much later in their life cycle.
The fact that the biases in the TC formation and tracks in the North Pacific are fairly similar among all model resolutions has prompted us to examine the large-scale environmental fields that determine the potential for tropical cyclogenesis. For this purpose, we compare the geographical distribution of the genesis potential index (GPI; see details of the definitions and calculations in Camargo et al. 2007b) computed from the ERA-40 reanalysis and the model data for the MJJASON season and the common period of 1990-2002 using monthly mean data (Fig. 2). The IFS has considerably higher values of GPI in the northwest Pacific between about $10^{\circ} \mathrm{N}$ and $25^{\circ} \mathrm{N}$ at all resolutions but more so at T2047. It is a consequence of the positive bias both in the potential intensity, midtropospheric relative humidity, and the lower-tropospheric vorticity and appears to be related to higher precipitation over the western tropical Pacific warm pool area in this model (see Fig. 1 in Dirmeyer et al. 2012). Vertical wind shear errors are small in the region where the bulk of cyclogenesis occurs and are positive (negative) to the south (north) from it. It is possible that excessive convective activity in this region could also lead to stronger TC activity. In the northeast Pacific, GPI on the other hand tends to have a negative bias over the area where TCs form. The dipole structure of the GPI bias in this region is related to the similar dipole structure in the vertical wind shear, midtropospheric humidity, and the lower-tropospheric vorticity, such that errors in these fields all contribute to the lower (higher) GPI to the north (south). It appears that these errors are associated with the quality of the model's intertropical convergence zone (ITCZ), which is weaker and slightly too confined to the equator (not shown). This model bias may affect the TC activity in the region, although other factors such as the Madden-Julian oscillation (MJO) and easterly wave activity could play a role as well (e.g., see Molinari and Vollaro 2000; Serra et al. 2010). Since the interaction between the orography and easterly waves influences cyclogenesis in the region (e.g., Zehnder et al. 1999), coarse representation of the Central American Mountains in the low-resolution simulations could be an additional factor responsible for the weak simulated TC activity in this basin.

In the North Indian Ocean, all versions of the model, except perhaps T159, display too much TC activity in the Bay of Bengal (Fig. 1, left panels) with the corresponding tracks crossing the northern part of the Indian subcontinent (Fig. 1, right panels) typical of monsoon depressions. The excessive number and intensity of the north Indian low pressure systems in IFS at all resolutions (see section 3) is likely a consequence of too strong convective activity in this basin (see Fig. 1 in Dirmeyer et al. 2012), similar to the northwest Pacific discussed above. 
a) OBS

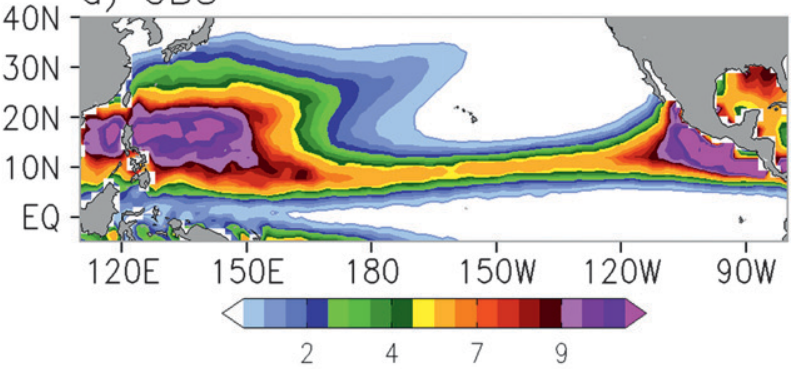

b) $\mathrm{T} 2047$ - OBS

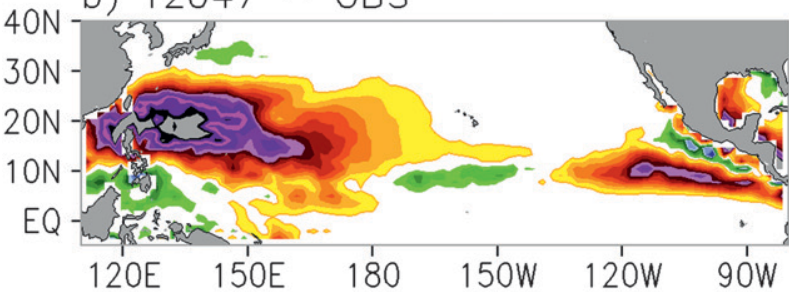

c) $T 1279-\mathrm{OBS}$

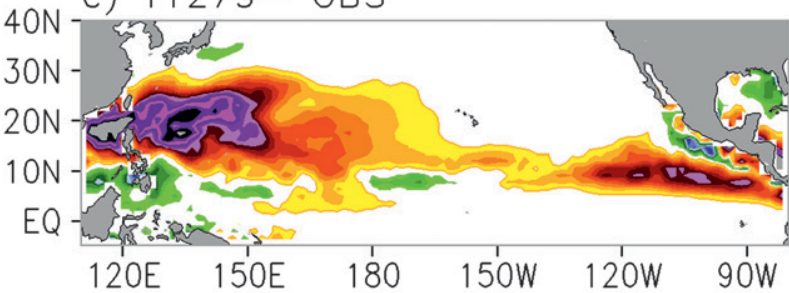

d) $T 511-\mathrm{OBS}$

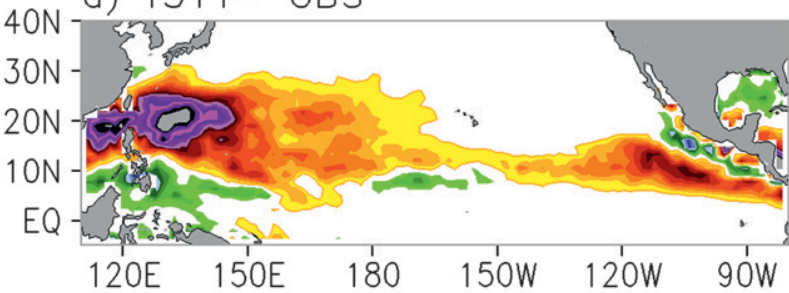

e) T159- OBS

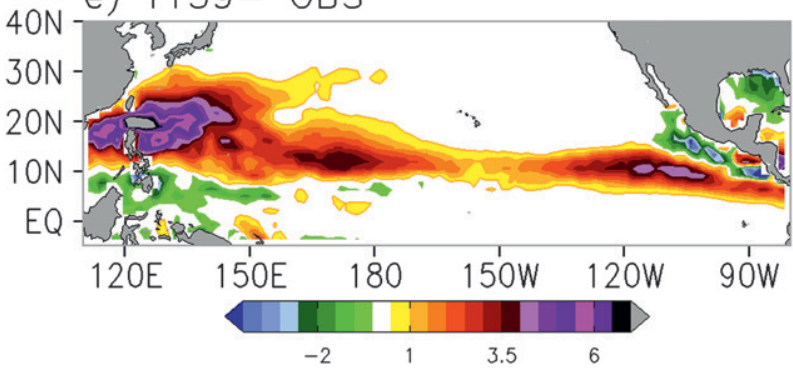

FIG. 2. MJJASON average GPI for the period 1990-2002 for (a) ERA-40 and IFS at (b) T2047, (c) T1279, (d) T511, and (e) T159 shown as deviations from the ERA-40 values.

The T159 simulation, which shows the most realistic TC frequency (see Table 2), has also the most realistic seasonal cycle in the basin (not shown) with a substantial decrease in the number of cyclones during the inactive season.

\section{b. North Atlantic}

The observed North Atlantic cyclogenesis is concentrated mainly in four regions: 1) the main development region (MDR) spanning approximately $10^{\circ}-20^{\circ} \mathrm{N}$ and $\left.30^{\circ}-85^{\circ} \mathrm{W}, 2\right)$ the Caribbean Sea, 3 ) the Gulf of Mexico, and 4) the western subtropical Atlantic (Fig. 3a). The corresponding track density is shown in Fig. $3 \mathrm{f}$ with the highest concentration of tracks in the central tropical Atlantic and along the eastern seaboard of North America. The simulated genesis density is generally lower, particularly at T159 and T511, reflecting the low TC count in the region; the details of its spatial distribution are simulated with different levels of success depending on the resolution of the model (Fig. 3, left panels). The modeltrack density shows systematic differences with the resolution change, as described in detail below, with the most realistic representation at the highest resolution of T2047 (Fig. 3, right panels)

Cyclogenesis in the MDR is fairly well defined in all model versions, although it is weaker than the observed, especially for the lowest resolution of T159. Activity over the African continent is likely due to the tropical easterly wave stage of the TC development (e.g., Thorncroft and Hodges 2001; Bengtsson et al. 2007a). Consequently, there are very few storms crossing the tropical Atlantic in the T159 model, with the track density becoming systematically more realistic in this region as the resolution is increased.

The Caribbean center of cyclogenesis is weak at T159 and is shifted to the northern tip of South America instead. The T159 model also produces too many slow moving storms in this region (filtered out in the present analysis-see section 3), possibly as a consequence of poorly resolved sharp orography in the northern Venezuelan highlands. The location and magnitude of the Caribbean center become more realistic at the higher resolutions, although it is overly strong at T2047.

The Gulf of Mexico center is not evident in the model simulations, which is largely a consequence of the displacement distance filtering (see section 3 ). The contribution of these systems to the total North Atlantic count is about 1-2 per season (not shown), with more storms at the higher resolutions. However, this is not expected to change the main conclusions regarding the TC frequency simulation in this basin and its sensitivity to model resolution (see section 4 and Table 2).

The western Atlantic region of cyclogenesis in the model is located more to the south/southeast from the observed one, and is excessively strong at T1279. At T2047, and to some extent at T1279, this region extends toward the continent, more in line with the observations. As a result, at the lowest three resolutions, most 

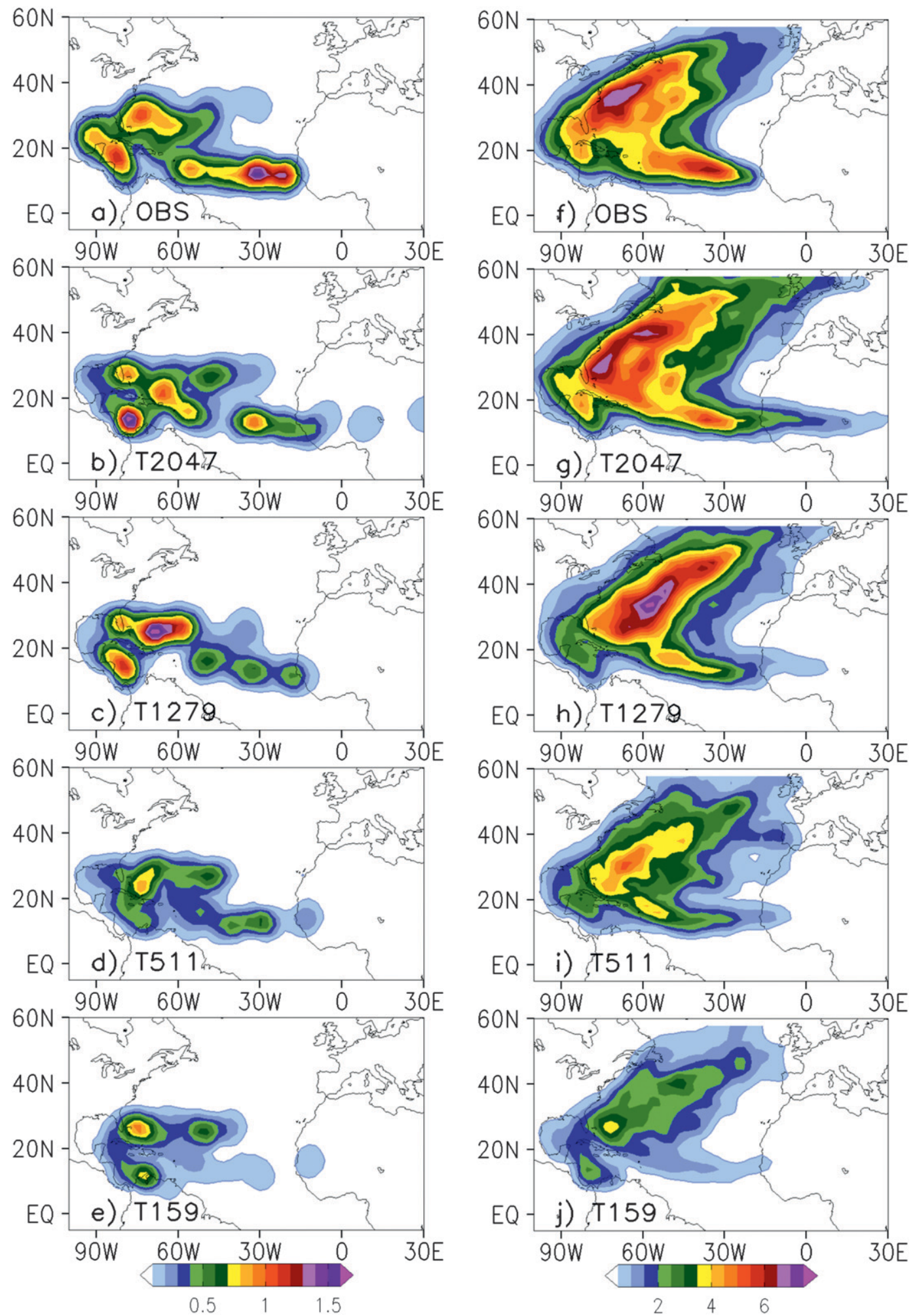

FIG. 3. As in Fig. 1, but for the North Atlantic. 
TCs in the subtropical Atlantic propagate parallel to but displaced away from the east coast of the United States, closer to the center of the basin. Only in the T2047 model, does the highest concentration of tracks occur close to the coast and have the magnitude comparable to the observed.

In summary, at the coarsest model resolution (T159), TC activity is low both in the MDR and the subtropical Atlantic (Fig. 3j). As the model resolution is increased, TC activity in the MDR strengthens and gradually grows in the subtropical Atlantic reaching realistic levels at T1279, although remaining well off the coast, as in the lower-resolution versions of the model (Figs. 3h,i,j). Similar results are obtained when the analysis period is extended to the longer 30-yr data (not shown). These results are also largely in agreement with the findings of Caron et al. (2010). As the resolution is increased further to T2047, a distinct change takes place in the distribution of the track density: more storms propagate closer to the east coast of the United States, in better agreement with observations, compared to all lower-resolution simulations examined (Fig. 3g). It would be of interest to determine the physical factors behind this change, though it is beyond the scope of the present paper. Contrary to the simulation of TC formation and tracks, model GPI in the North Atlantic is fairly similar among different resolutions (not shown). It has a positive bias in the MDR, likely related to the excessive rainfall in the western Atlantic (see Fig. 1 in Dirmeyer et al. 2012), and negative biases off the coast of Florida, in the Gulf of Mexico, and off the coast of West Africa. The latter biases have somewhat larger aerial extent in the T159 simulation.

\section{Intensity distribution}

The TC intensity is generally represented by the lifetime maximum surface wind speed and/or minimum central SLP (e.g., Wang and Wu 2004). In this section, we present distributions of both quantities for all four resolutions and different ocean basins including the windpressure relationship for the $\mathrm{NH}$.

\section{a. Maximum surface wind speed}

As has been mentioned earlier, TC intensity critically depends on the model resolution, among other factors. The question is how much further improvement in the simulation of the TC intensity distribution can be achieved by moving from a low AGCM resolution of, for example, $100 \mathrm{~km}$ to resolutions of 16 or $10 \mathrm{~km}$, while retaining conventional parameterizations, such as the one for cumulus convection.

Figure 4 shows the frequency of occurrence of maximum 10-m wind speed for the $\mathrm{NH}$ and separately for the northwest Pacific, the northeast Pacific, and the North Atlantic. There is a qualitative difference between the distributions for the T159 and T511 cases on the one hand, and the T1279 and T2047 on the other, independent of the ocean basins examined: the distributions are too narrow and skewed toward lower wind speeds, and their right tails, including secondary peaks, are not reproduced at all at the lower two resolutions. For T1279, the shape of the distribution in the higher wind speed range (beyond $35 \mathrm{~m} \mathrm{~s}^{-1}$ ) becomes more similar to that observed. As the resolution is increased further to T2047, the distribution is improved in this range, although there are no changes at the extreme end except perhaps in the North Atlantic (Fig. 4d). As a result, storms at T2047 appear to be stronger on average than at T1279, which is also consistent with TC forecasting studies (e.g., Shen et al. 2006; Fierro et al. 2009).

In addition to the direct effect of the resolution on the TC intensity, other factors that could influence the simulation of the intensity distribution are as follows: 1) duration of storms, as longer-lived storms have more time to intensify; 2) intensification rate; and 3) underlying SSTs (storms developing over warmer SSTs could become more intense). We have found that the lowerresolution storms have on average less time to intensify, based on the sample equal to the quarter of the most intense storms at each resolution (not shown). This is particularly evident for the T159 simulation, where the average TC intensification time is significantly shorter compared to the T1279 and T2047 simulations. Analysis in section 6 a.4 also suggests that the lower-resolution storms have additionally a weaker intensification rate. Regarding the underlying SSTs, we do not see any clear systematic differences among the model resolutions.

We also find significant improvement in the simulation of the most intense TCs relative to some recent studies (e.g., Oouchi et al. 2006; Zhao et al. 2009; Caron et al. 2010). The highest simulated surface wind speeds are 68.4 (T2047), 65.2 (T1279), 46.4 (T511), and 29.9 $\mathrm{m} \mathrm{s}^{-1}$ (T159), all occurring in the northwest Pacific. In this basin, T1279 and T2047 integrations also tend to produce stronger storms than observed (Fig. 4b). For the extended period of analysis (1979-2008), the highest simulated wind speed is $66.4 \mathrm{~m} \mathrm{~s}^{-1}$ for the T1279 resolution. Thus, the T1279 and T2047 results exceed the current "record" of $55 \mathrm{~m} \mathrm{~s}^{-1}$ obtained by Oouchi et al. (2006) by a significant margin, although in their study, the duration of simulation was shorter, and the SST forcing was constructed by averaging the observed SST from 1982 to 1993. In the North Atlantic, the maximum surface wind speed is $56.6 \mathrm{~m} \mathrm{~s}^{-1}$ (T2047), which is characteristic of a category-4 hurricane. So, even at the highest model resolution, the most intense storms observed in nature 

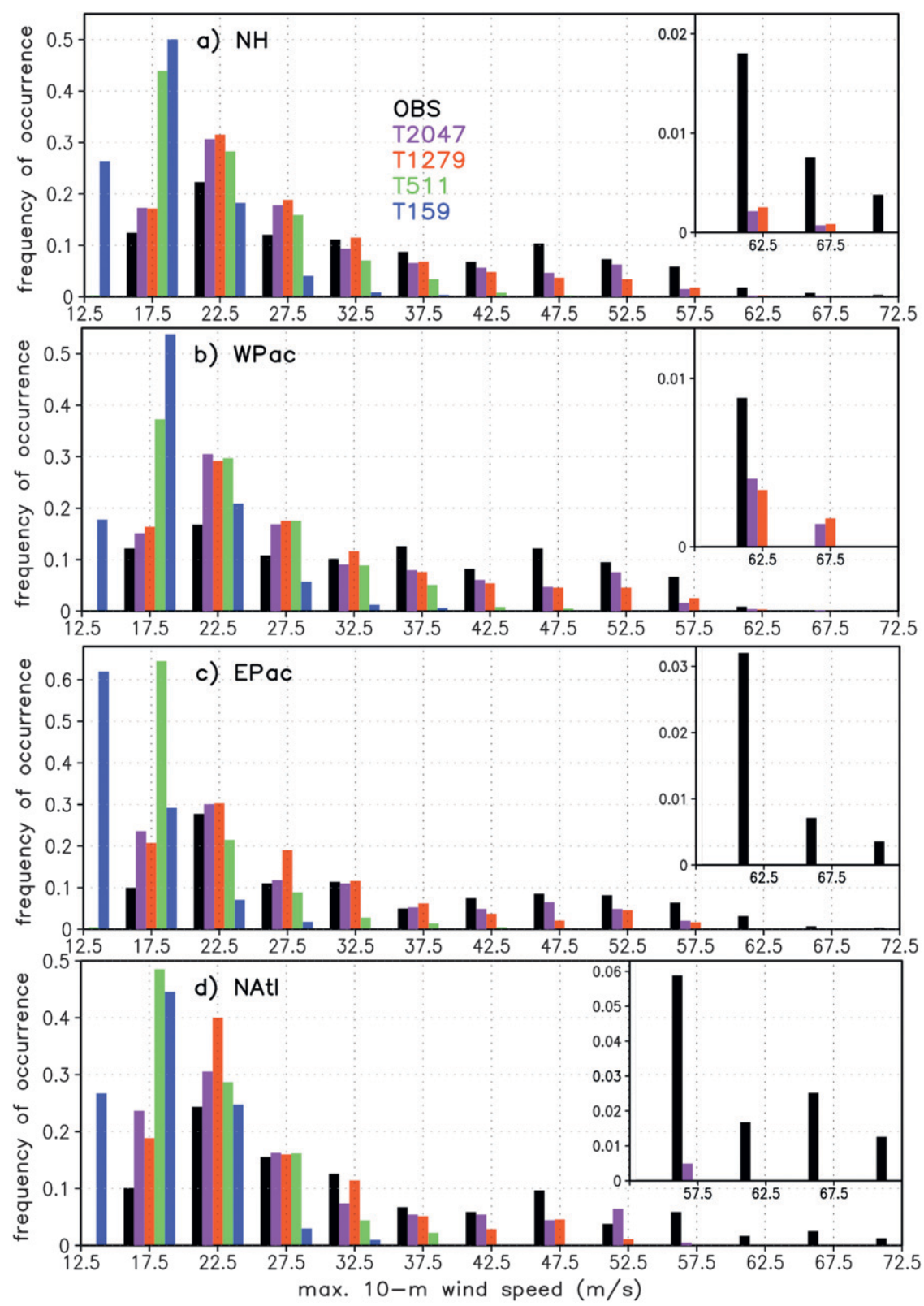

FIG. 4. Distribution of maximum attained 10-m wind speed in the (a) Northern Hemisphere, (b) northwest Pacific, (c) northeast Pacific, and (d) North Atlantic TCs from the IBTrACS data (black bar), IFS T2047 (purple bar), T1279 (red bar), T511 (green bar), and T159 (blue bar) for MJJASON of 1990-2008. Inset plots show the tail of the distributions.

(category 5) are not simulated. This could be expected since the $10-\mathrm{km}$ grid spacing is still too coarse to adequately resolve the near-eye structure.

\section{b. Minimum sea level pressure}

The frequency of occurrence of minimum SLP is shown in Fig. 5. The main conclusions reached in the previous sections regarding the distributions of the maximum surface wind speed would also apply for this variable.
There are, however, some important differences. The T1279 and T2047 distributions are much more similar for the minimum SLP than the maximum wind speed, except perhaps in the northeast Pacific and the North Atlantic, especially in the left tail of the distributions. At the T1279 and T2047 resolutions, the model is capable of generating minimum SLP considerably lower than what is suggested by the observations, particularly in the Pacific (Figs. 5b,c). In the North Atlantic, the 

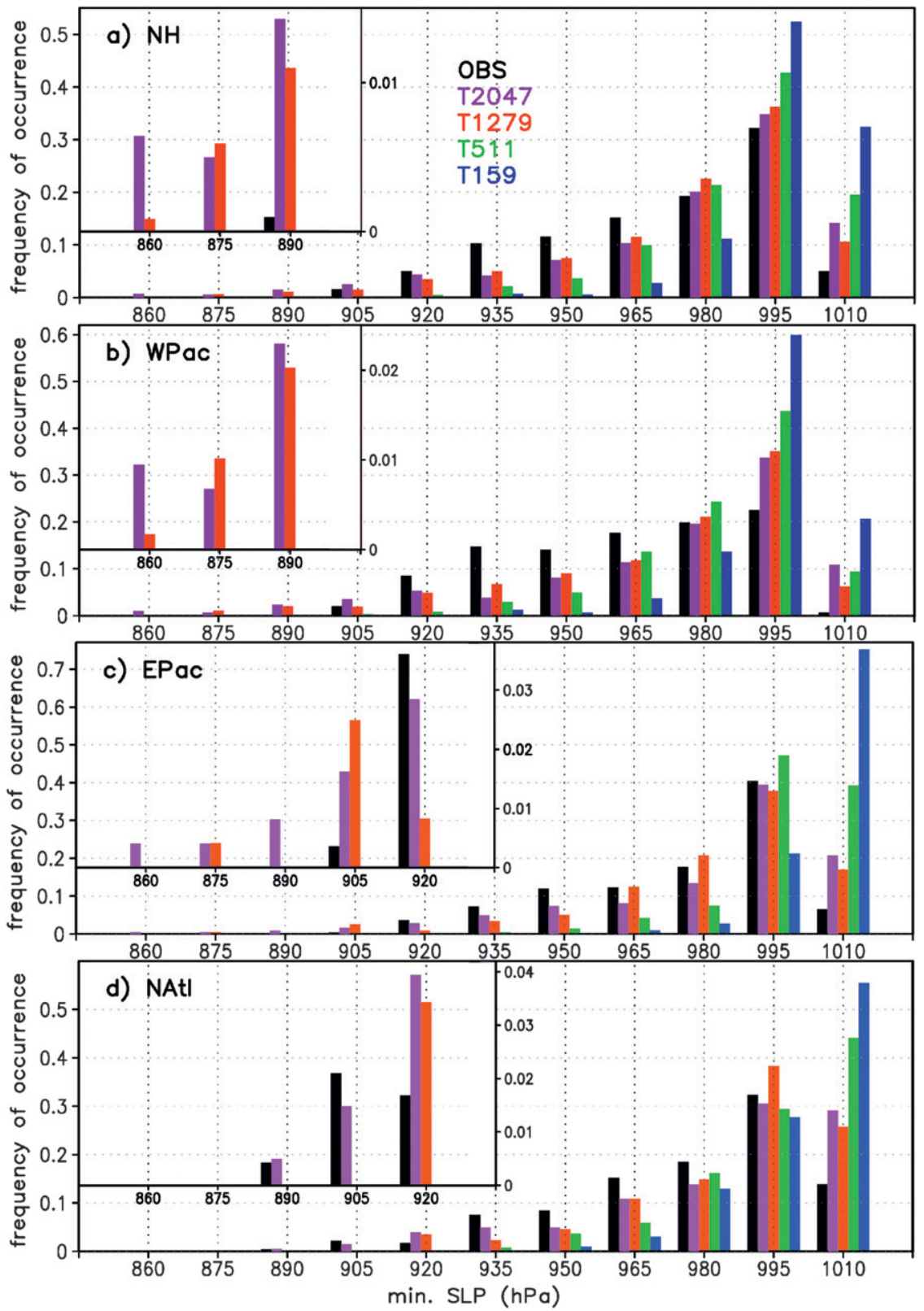

FIG. 5. As in Fig. 4, but for minimum SLP.

simulated minimum SLP is close but does not get lower than the observational estimate (Fig. 5d). In this basin, the most intense storm simulated by the model in fact reaches a central pressure of $885 \mathrm{hPa}$ (T2047), corresponding to a category-5 hurricane, comparable to the record low of $882 \mathrm{hPa}$ of Hurricane Wilma in 2005.

If we consider SLP as a measure of the TC intensity, then simulations at the highest two resolutions could be considered very good, except that occasionally the model produces overly strong TCs. What could be the reason for this discrepancy? One possibility is the absence of an interactive ocean. It has been shown in a number of studies (see Emanuel 2003 and the references therein) that the SST cooling induced by a TC has a dominant (weakening) effect on its intensity. Nevertheless, these results demonstrate that with a model resolution of 10 or $16 \mathrm{~km}$, it is possible to generate TCs with warm-core magnitudes (see section 6a.2) and consequently minimum SLP values characteristic of very intense storms found in nature.

\section{c. Wind-pressure relationship}

Another way to assess the quality of the TC intensity simulation is by means of displaying maximum surface 


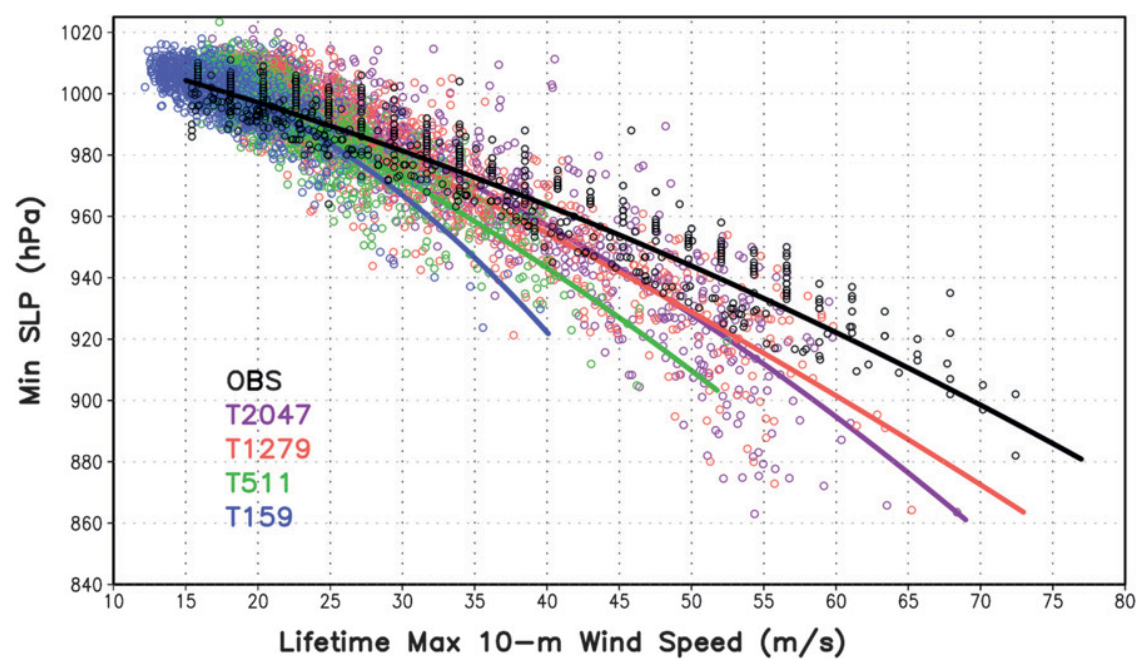

FIG. 6. Scatter diagram of the lifetime maximum $10-\mathrm{m}$ wind speed $\left(\mathrm{m} \mathrm{s}^{-1}\right)$ vs corresponding central SLP (hPa) for observed (IBTrACS; black) and IFS T2047 (purple), T1279 (red), T511 (green), and T159 (blue) Northern Hemisphere TCs for MJJASON of 1990-2008. Solid lines of the corresponding colors show the second-order polynomial fit to the data points.

wind speed with respect to the corresponding central SLP. This wind-pressure relationship is shown in Fig. 6 for the whole $\mathrm{NH}$ along with the lines denoting the second-order polynomial fit to the data points in the scatter diagram. This choice of the fitting function is consistent with the gradient wind balance (e.g., Knaff and Zehr 2007). The following conclusions are supported by Fig. 6. 1) Independent of the resolution, the model has difficulty generating strong enough wind speeds for a given central pressure. This seems to be a universal problem with current models (Knutson et al. 2007; Walsh 2008; Caron et al. 2010) and has been attributed to insufficient resolution. Indeed, Fig. 6 shows that with the increase of resolution, this deficiency is systematically reduced, with the possible exception of the T2047 case that is addressed below. At low SLP, this problem could also be related to the deficiencies in the surface momentum flux parameterization, namely, the surface roughness being too high in the high wind speed range (e.g., Powell et al. 2003; Liou 2007). 2) At T1279 and T2047, the model performs quite well down to about $960 \mathrm{hPa}$. Below this value, there is a sharp drop in the simulated maximum wind speed, which becomes worse at lower SLP. This behavior is similar to the results of Oouchi et al. (2006; their Fig. 5, left panel). Since the T2047 model simulates more storms with maximum intensity in the range of 940 to $860 \mathrm{hPa}$, the fit for this case indicates a somewhat worse performance compared to T1279. Most importantly, there is no improvement in the simulation of the wind-pressure relationship for the very intense storms as the resolution is increased further from T1279 to T2047. One perhaps needs to go to very fine resolutions on the order of $1 \mathrm{~km}$ and explicit convection, as well as more realistic surface roughness parameterization, to capture the observed relationship.

\section{Structure of the tropical cyclones}

\section{a. Structure of the TC composites}

\section{1) Compositing methodology}

To compare the TC structure across several model resolutions, we formed composites of the most intense TCs following the methodology described in Bengtsson et al. (2007b) and Catto et al. (2010). For each model resolution, we average five of the most intense storms (in terms of the $10-\mathrm{m}$ wind speed) at the time when they achieve their maximum intensity, as indicated by the lifetime maximum value of the $10-\mathrm{m}$ wind speed. The number of storms in the composite is partly limited by the sharp increase in computing and storage requirements for processing data at the highest model resolutions. In addition, since the emphasis is on the most extreme storms, their number will always be small in such a short integration. Longer simulations at these resolutions are needed to increase statistical robustness when comparing extremes. However, examination of individual storms in each composite (not shown) revealed a large degree of similarity between the selected systems, at least in terms of the quantities presented in Figs. 7 and 8 . This could be related to the fact that we are sampling the most intense storms (for each model simulation) of quite similar intensities (see below). Some of the structural parameters, 

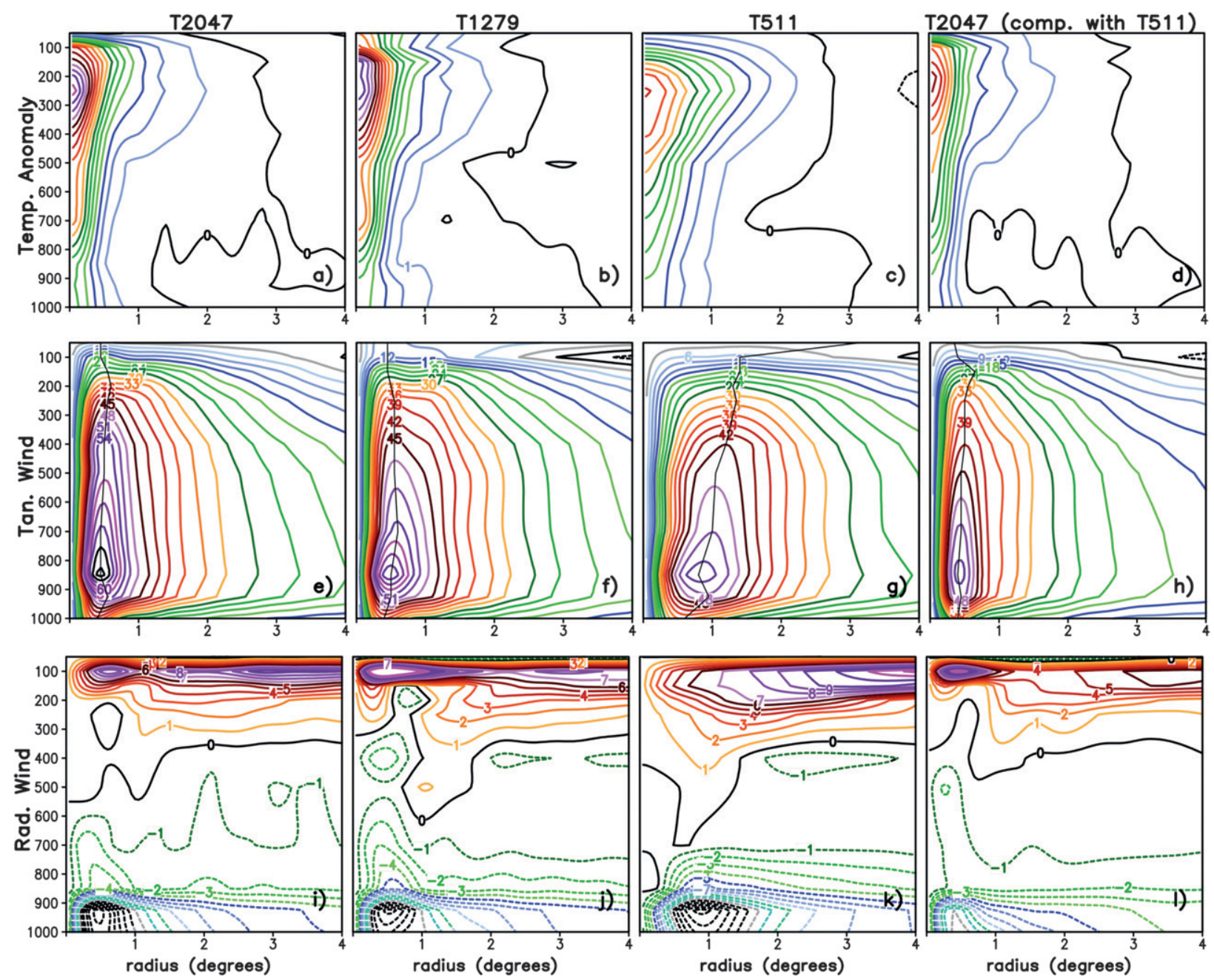

FIG. 7. Azimuthally averaged (top) temperature anomaly, (middle) tangential wind, and (bottom) radial wind for the composite of the five most intense storms at (a),(e),(i) T2047; (b),(f),(j) T1279; and (c),(g),(k) T511. (d),(h),(l) The T2047 composite of five storms with intensities similar to the T511 composite (see text for more details) is shown. The thin black line in (e)-(h) connects maximum velocity at each level and indicates the vertical tilt of RMW. Temperature units are $\mathrm{K}$. Wind units are $\mathrm{m} \mathrm{s}^{-1}$. Contour interval is $1 \mathrm{~K}$ for temperature, $3 \mathrm{~m} \mathrm{~s}^{-1}$ for tangential wind, and $1 \mathrm{~m} \mathrm{~s}^{-1}$ for radial wind. Negative contours are dashed.

like the radius of maximum wind (RMW), are known to be quite comparable for such TCs (e.g., Bell and Sawin Ray 2004; Mallen et al. 2005).

The storms are selected from the northwest Pacific, which is the domain where the most intense TCs are likely to take place (Fig. 4b). The occurrence of maximum intensity is restricted to the south of $30^{\circ} \mathrm{N}$ to avoid any influences of extratropical transition. Sampling of the fields associated with a propagating storm is performed in a radial coordinate system centered on the storm center (details in the appendix of Bengtsson et al. 2007b). Prior to compositing, the radial grid is oriented with the direction of storm propagation to reduce the influences of storm direction on the composite structure. At each pressure level, the quantities to composite are extracted with respect to the storm center defined as a maximum in the T511-truncated vorticity at the same level. This is found to significantly improve the accuracy of the composites compared to the use of N80-truncated vorticity to define the storm center. These maxima are computed by referencing tracks to the T511 resolution vorticity field at each level using steepest ascent maximization as before. Surface and column-integrated quantities are extracted with respect to the T511 vorticity maximum at $1000 \mathrm{hPa}$.

The characteristics of the five most intense northwest Pacific TCs are shown in Table 3. The range of the maximum surface wind speed and concurrent minimum SLP attained by these storms in the T2047 simulation places them practically in the supertyphoon category 
Tangential Wind at $850 \mathrm{hPa}$
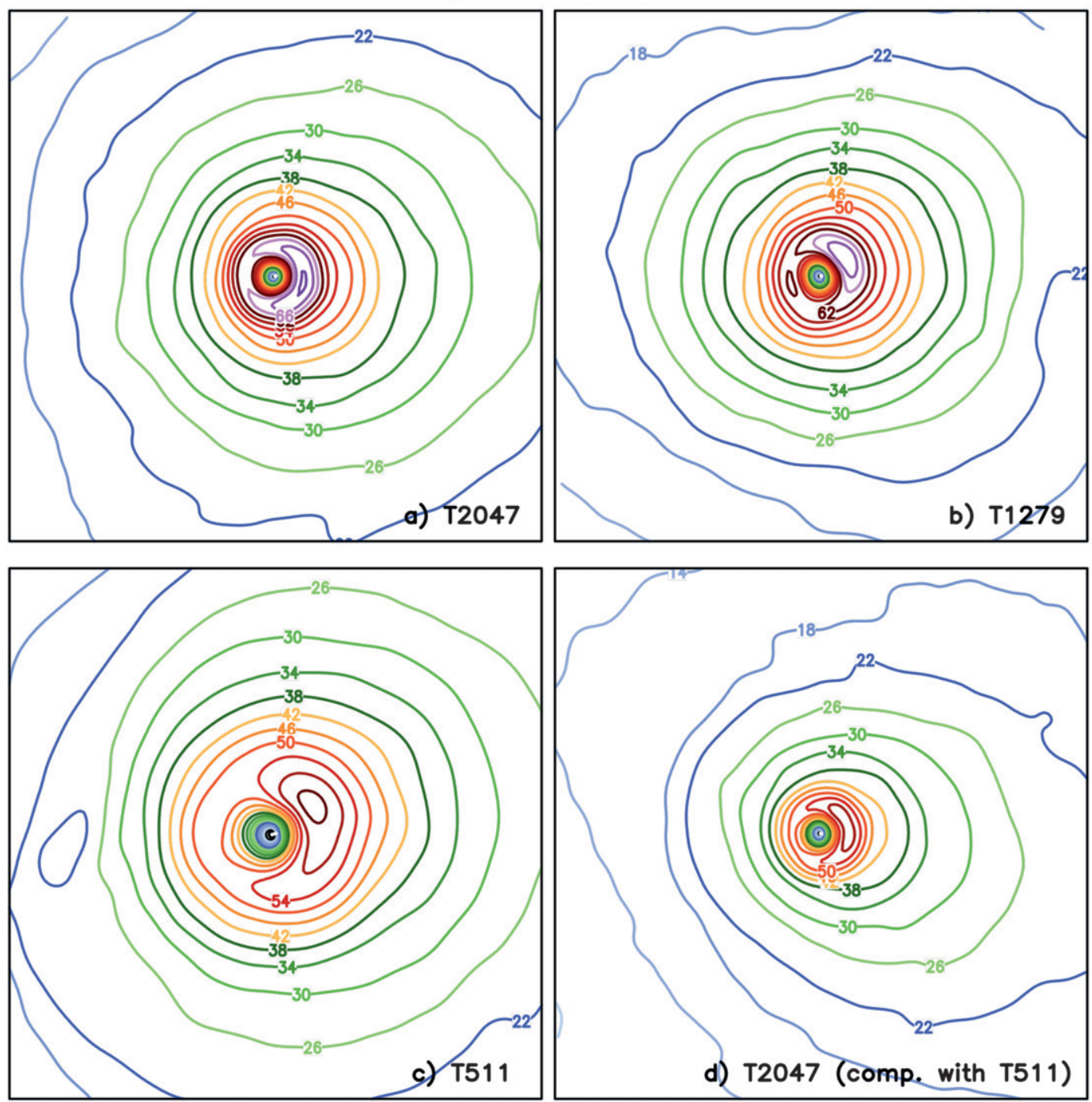

FIG. 8. Tangential wind at $850 \mathrm{hPa}$ for the horizontal composite of the five most intense storms at (a) T2047, (b) T1279, and (c) T511 in a radial coordinate system centered on the storm center. (d) The T2047 composite of five storms with similar intensities to the T511 composite (see text for more details) is shown. Radius is $4^{\circ}$. Units are $\mathrm{m} \mathrm{s}^{-1}$. Contour interval is $4 \mathrm{~m} \mathrm{~s}^{-1}$.

(e.g., Weatherford and Gray 1988a). The latitudes of maximum intensity are also consistent with Weatherford and Gray (1988a). The T1279 systems are somewhat weaker compared to T2047 but also belong to the category of supertyphoons. For the T511 case, the most intense storms are considerably weaker than both the T2047 and the T1279 counterparts and belong to the broad category of typhoons.

TABLE 3. Characteristics of the composited TCs. Shown are range and average (in the parenthesis) of the selected quantities.

\begin{tabular}{|c|c|c|c|c|}
\hline & \multicolumn{3}{|c|}{ Composite of the most intense storms } & \multirow{2}{*}{$\begin{array}{c}\begin{array}{c}\text { Composite of storms } \\
\text { similar to T511 }\end{array} \\
\text { T2047 }\end{array}$} \\
\hline & T2047 & T1279 & T511 & \\
\hline Maximum surface wind speed, $\mathrm{m} \mathrm{s}^{-1}$ & $59.8-68.4(62.6)$ & $59.0-65.2(61.7)$ & $43.0-46.4(44.9)$ & $42.9-46.9(44.7)$ \\
\hline Concurrent minimum SLP, hPa & $921-864(886)$ & $927-864(900)$ & $937-905(922)$ & $934-917(925)$ \\
\hline Latitude of maximum intensity & $11^{\circ}-24^{\circ} \mathrm{N}\left(18^{\circ} \mathrm{N}\right)$ & $18^{\circ}-23^{\circ} \mathrm{N}\left(20^{\circ} \mathrm{N}\right)$ & $18^{\circ}-22^{\circ} \mathrm{N}\left(20^{\circ} \mathrm{N}\right)$ & $17^{\circ}-26^{\circ} \mathrm{N}\left(22^{\circ} \mathrm{N}\right)$ \\
\hline
\end{tabular}


The same analysis was attempted on the T159 data, but the procedure outlined above did not work in the same way for this simulation as for the other three. First, $\sim 25$ of the most intense northwest Pacific storms in the T159 case attain their peak surface wind speed much farther north of the $30^{\circ} \mathrm{N}$ latitude and are visibly affected by the extratropical transition. Second, the strongest five systems south of $30^{\circ} \mathrm{N}$ all happen to intensify close to the land areas. The warm core and horizontal wind structures of these TCs exhibit features that are likely caused by the proximity to land (not shown), and they have relatively larger sizes compared to the other three simulations (the inner core is wider and more diffuse). For the T159 simulation, the number of TCs in the composite would probably need to be much larger to average out these influences, or it may be necessary to choose other criteria for compositing, such as a low-level vorticity maximum. For these reasons, we do not include the T159 results in the present comparison.

\section{2) Vertical STRUCTURE}

Figure 7 shows the vertical profiles of the azimuthally averaged composites of the temperature anomaly (relative to the mean temperature over a $5^{\circ}$ radius for all resolutions), and the tangential and radial winds for the T2047, T1279, and T511 simulations. For computational efficiency multilevel (pressure level) variables are truncated to the resolution of T511, common to all three simulations, prior to the regional sampling. This resolution should be sufficient to determine the large-scale structure of the TC composites and compare it among the simulations, although the peak values of the variables shown in Figs. 7 and 8 could be somewhat underrepresented for the T1279 and T2047 models. The compositing is done on 11 pressure levels between 1000 and $50 \mathrm{hPa}$. The radial grid extends to $5^{\circ}$ (geodesic) with $0.05^{\circ}\left(0.1^{\circ}\right)$ resolution in the radial (azimuthal) direction. All three models simulate a distinct warm core throughout the troposphere with a maximum anomaly at the 250-hPa level (Figs. 7a-c) consistent with the observational study of Frank (1977) and a number of lower-resolution modeling studies (e.g., Bengtsson et al. 1995; Bengtsson et al. 2007b; LaRow et al. 2008). As the model resolution is increased, TC intensity has a potential to increase because of the better-resolved pressure gradients in the inner core. This would lead to stronger moisture flux at the surface, increased moisture convergence in the boundary layer, and enhanced latent heat flux release throughout the troposphere leading to the intensification of the warm core. Moisture convergence could also increase because of a better-resolved secondary circulation. As a result, maximum temperature anomaly increases from $11.1 \mathrm{~K}$ at $\mathrm{T} 511$ to $16.8 \mathrm{~K}$ at
T1279 and to $17.5 \mathrm{~K}$ at T2047. The latter two values are quite comparable to the observed mean maximum temperature anomaly of more than $15^{\circ} \mathrm{C}$ for northwest Pacific typhoons (Frank 1977), although it is obtained by averaging data from more than 100 storms. In addition, the warm core becomes more compact at the higher resolutions and, as a result, has stronger radial temperature gradients.

The azimuthally averaged tangential winds (Figs. 7e-g) show cyclonic flow throughout the troposphere (within the inner core, or about 2 degrees from the composite center in the model) with the maximum at $850 \mathrm{hPa}$ and the appearance of anticyclonic flow in the upper troposphere outside the inner core, all in line with observations (Frank 1977). With the increase of resolution, the eyewall (region of maximum tangential winds) has a tendency to become narrower; the maximum wind increases from $55.9 \mathrm{~m} \mathrm{~s}^{-1}$ at T511 to $65.6 \mathrm{~m} \mathrm{~s}^{-1}$ at T1279 and to $70.0 \mathrm{~m} \mathrm{~s}^{-1}$ at T2047. As a result, the eyewall is defined by stronger gradients at the higher resolutions. The RMW (at $850 \mathrm{hPa}$ ) decreases from $0.75^{\circ}$ at $\mathrm{T} 511$ to $0.45^{\circ}$ at $\mathrm{T} 1279$ and to $0.4^{\circ}$ at T2047. Although RMW tends to be smaller for more intense TCs (Weatherford and Gray 1988b; Bell and Sawin Ray 2004; Mallen et al. 2005), the difference between the T511 and the T1279/ T2047 RMW appears to be due to differences in the model resolution, as is shown below. In agreement with the smaller RMW, the eyewall slopes less with height, as shown by the thin black lines in Figs. 7e-g (Stern and Nolan 2009). In addition, winds in the eyewall at T2047 do not decay as rapidly with height as at the lower resolutions, which is also one of the observed features of TCs (Stern and Nolan 2009).

The azimuthally averaged radial winds (Figs. 7i-k) display the well-observed inflow in the lower troposphere and the outflow in the upper troposphere (Frank 1977). The systematic change of the largest inflow and outflow with the resolution is less clear: the extreme values of inflow (outflow) are -16.3 (13.6), -15.3 (18.5), and $-17.4 \mathrm{~m} \mathrm{~s}^{-1}\left(10.6 \mathrm{~m} \mathrm{~s}^{-1}\right)$ for T511, T1279, and T2047, respectively. At the highest two resolutions, the inflow and outflow within the inner core are largely confined to below $800 \mathrm{hPa}$ and above $300 \mathrm{hPa}$, respectively, as observed (Frank 1977). In contrast, the T511 radial wind distribution in this region is more diffuse in the vertical. Also, there is a local upper-level maximum of outflow within the inner core at T1279 and T2047, which is not present at T511. At this point, it is not clear whether this is just a model artifact or has some relevance to reality. In summary, the vertical structure of the most intense TCs becomes much more realistic at T1279 and T2047 and appears to be a marked improvement compared to previous climate simulations (Bengtsson et al. 1995; LaRow et al. 2008; Caron et al. 2010). 


\section{3) Horizontal structure}

Figure 8 shows horizontal composites of the tangential winds at $850 \mathrm{hPa}$ for the T2047, T1279, and T511 simulations. The radial grid extends to $4^{\circ}$ (geodesic) in this figure and has a $0.05^{\circ}\left(0.1^{\circ}\right)$ resolution in the radial (azimuthal) direction. The display is oriented so that the direction of propagation of the composited storms is due north. We show the horizontal winds at $850 \mathrm{hPa}$ rather than 10-m level because of the presence of strong mesoscale features and large spatial variability in the latter for the highest model resolutions (see Fig. 10), which leads to the reduction in quality of the corresponding composites. The winds in Figs. 8a-c show a clear wavenumber-1 asymmetry with a maximum to the east-northeast of the storm center, in general agreement with the observational studies of Frank (1977), Kepert (2006a), and Kepert (2006b). For the T2047 and T1279 cases, the wind distributions are quite similar: maximum winds are 74.5 and $74.0 \mathrm{~m} \mathrm{~s}^{-1}$, respectively, both occurring at $0.4^{\circ}$ from the composite storm center. At T511, the wind maximum is $63.6 \mathrm{~m} \mathrm{~s}^{-1}$, its distance from the center is $0.65^{\circ}$, and the eyewall becomes visibly more diffuse than in the higher-resolution simulations.

The main goal of the TC composite analysis above is to assess the three-dimensional structure of the most intense TCs that each model is capable of simulating. It is understood that some differences could be related to the differing intensities of the sampled storms, in addition to the model resolution. To assess the impact of resolution on the above results, we selected five storms from the T2047 simulation that had similar intensities to the T511 storms (see Table 3). The corresponding structure of this different composite is shown in Figs. 7d, 7h, 7l, and $8 \mathrm{~d}$. The maximum temperature anomaly is $12.4 \mathrm{~K}$, maximum azimuthally averaged and $850-\mathrm{hPa}$ tangential wind is 55.1 and $60.2 \mathrm{~m} \mathrm{~s}^{-1}$, respectively. While these peak magnitudes are similar to the T511 counterparts, the structural differences between the two composites are large (cf. Figs. 7c,g,k with Figs. 7d,h,l and Fig. 8c with Fig. 8d). In fact, the structural parameters of the different T2047 composite (e.g., radial extent of the warm core, RMW and its vertical tilt with height, depth of inflow and outflow layers) are quite similar to the original T2047 composite (Figs. 7a,e,i and Fig. 8a). These results support previous findings (e.g., Bengtsson et al. 2007b; Caron et al. 2010) that model resolution may not only limit the peak intensities of simulated TCs but also their structural realism.

\section{4) INTENSITY LIFE CYCLE}

In addition to the above analysis of the threedimensional structure of the most intense TCs at different model resolutions, here we perform comparison of the temporal evolution of the composited storms. For this purpose, we examine intensity life cycles (both in terms of the maximum 10-m wind speed and minimum SLP) of the composites described in section 6a.1., except that the number of storms in each case is extended to 25 to reduce noisiness of the results. Model life cycles are compared with the ones from observations based on the composite of the 25 most intense typhoons in the northwest Pacific in the IBTrACS dataset (Fig. 9). To construct the life cycle composites, each storm is centered on the time when it achieves maximum intensity in terms of the $10-\mathrm{m}$ wind speed.

It is evident that model storms do not grow as fast as the observed ones, particularly at the lower resolution of T511, as witnessed in both the surface winds (Fig. 9a) and SLP (Fig. 9b). The reason for the curious spike (drop) in the surface wind speed six hours before (after) the peak intensity is not clear. The growth also starts earlier in the life cycle in all three models compared to observations. These model discrepancies could be related to the insufficient resolution and the choice of physical parameterizations (surface and boundary layer, convection, and microphysics), as some important internal processes and large-scale atmospheric influences may not be resolved or simulated (Wang and Wu 2004; Gopalakrishnan et al. 2011). The interaction with the warm ocean features such as warm eddies is also a known factor in the rapid intensification and peak intensity maintenance of the observed northwest Pacific typhoons (e.g., Wu et al. 2007; Lin et al. 2008). However, the absence of the atmosphere-ocean feedback in the current experimental setup is expected on the contrary to exaggerate the oceanic influence (see Fig. 2 in Wu et al. 2007).

It is curious that apart from the initial sharp drop in intensity, the rate of weakening of surface winds, at least during the first 10 time steps, and the filling of SLP in the $10-\mathrm{km}$ model is comparable to the observed, in contrast to the 16- and 39-km simulations. Figure 9 also shows a comparable increase in intensity at 10- and 16-km resolutions, but the decay is quite different between these models. It is not exactly clear what causes these differences, but the influence of sampling cannot be entirely ruled out. The decaying stage of the life cycle could be sensitive to the choice of the storms, particularly their location when they are likely to be recurving into the extratropics, and the large-scale environment they are moving into. As mentioned above, ultimately longer simulations are needed to increase the number of the most intense storms to be sampled, which would make composites statistically more robust.

\section{b. Surface snapshots of the intense TCs}

One of the main objectives of the numerical simulation of TCs is their realistic representation at the surface 

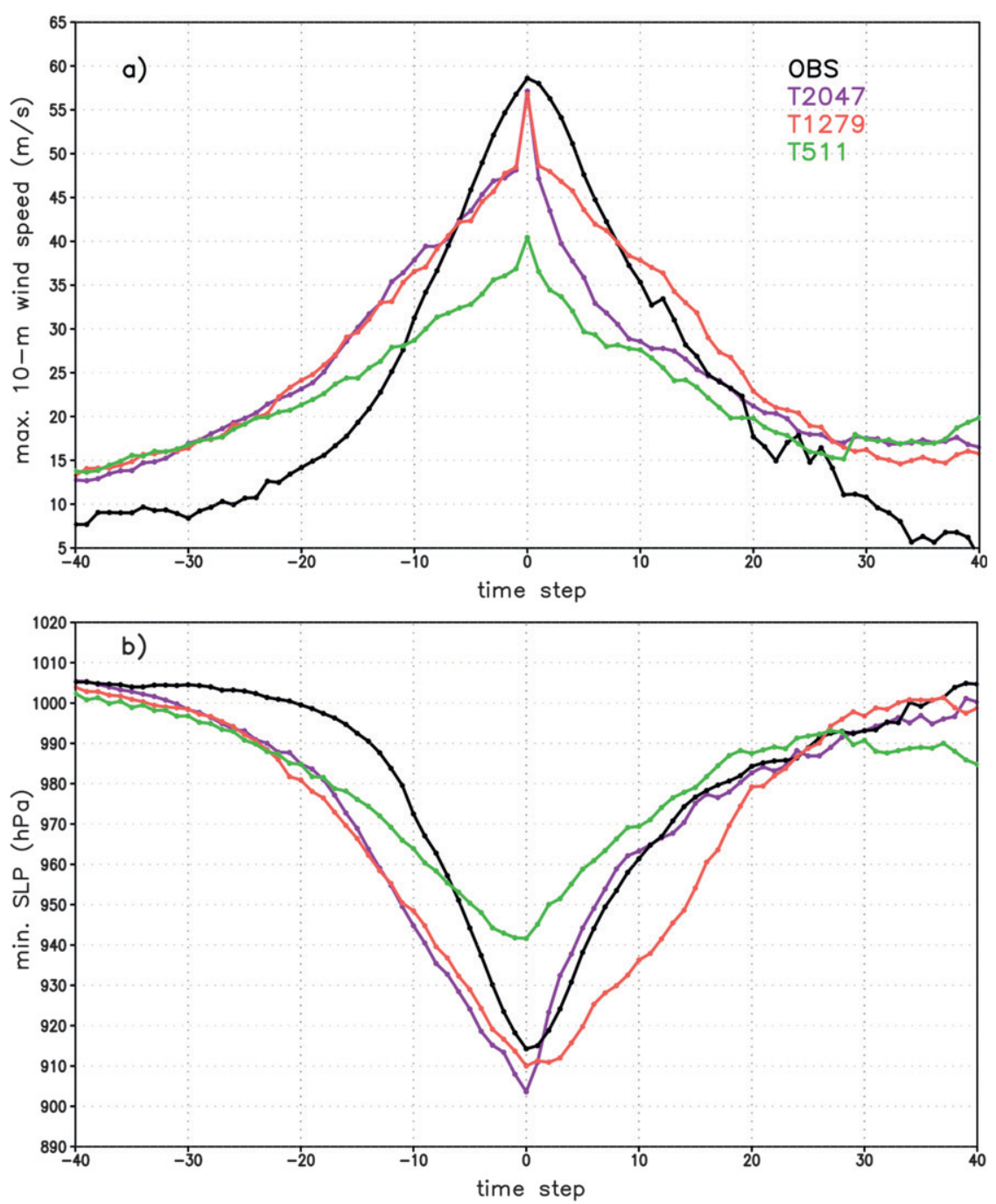

FIG. 9. Life cycle composite of (a) the maximum 10-m wind speed and (b) the minimum SLP based on the 25 most intense typhoons, in terms of the maximum 10-m wind speed, in the northwest Pacific for IBTrACS data (black), IFS T2047 (purple), T1279 (red), and T511 (green) during MJJASON of 1990-2008. Time step is in 6-h increments.

since this is where most damage from these systems occurs. Here, we present a surface snapshot of some of the most intense TCs, simulated by the IFS at the highest three resolutions, at the peak of their intensity. Figure 10 shows distributions of $10-\mathrm{m}$ tangential wind and the total column liquid and ice water (TCLIW), which are sampled at their native resolution. The radial grid extends to $2^{\circ}$ in this figure and has a $0.05^{\circ}\left(0.1^{\circ}\right)$ resolution in the radial (azimuthal) direction. As before, the display is oriented so that the direction of propagation of the storms is due north. The T2047 and T1279 surface wind varies on a smaller scale and has greater detail in the inner core, as well as more intense gradients in the eyewall, compared to the T511 simulation (Figs. 10a-c), which is more in line with the very high resolution limited area simulations (e.g., Chen et al. 2007). The T2047 storm is also quite axisymmetric, which is typical of the intense TCs. In both T2047 and T1279 cases, the maximum tangential wind is about $56 \mathrm{~m} \mathrm{~s}^{-1}$ and the RMW is $0.25^{\circ}$ or roughly $27 \mathrm{~km}$, in excellent agreement with the observed median RMW of $27.8 \mathrm{~km}$ for the most intense TCs (Kimball and Mulekar 2004). ${ }^{3}$ In contrast, the T511 maximum wind is $45.8 \mathrm{~m} \mathrm{~s}^{-1}$ and

\footnotetext{
${ }^{3}$ RMW is defined here as the radius of the maximum azimuthally averaged $10-\mathrm{m}$ tangential wind. The same results are obtained if the 10-m wind speed is used instead, which directly corresponds to the definition of RMW in the observational datasets (e.g., Kimball and Mulekar 2004).
} 


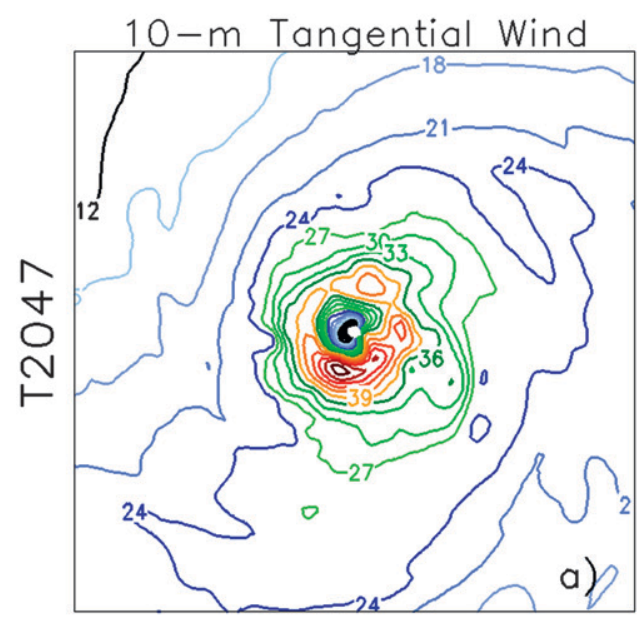

Total Column Liquid
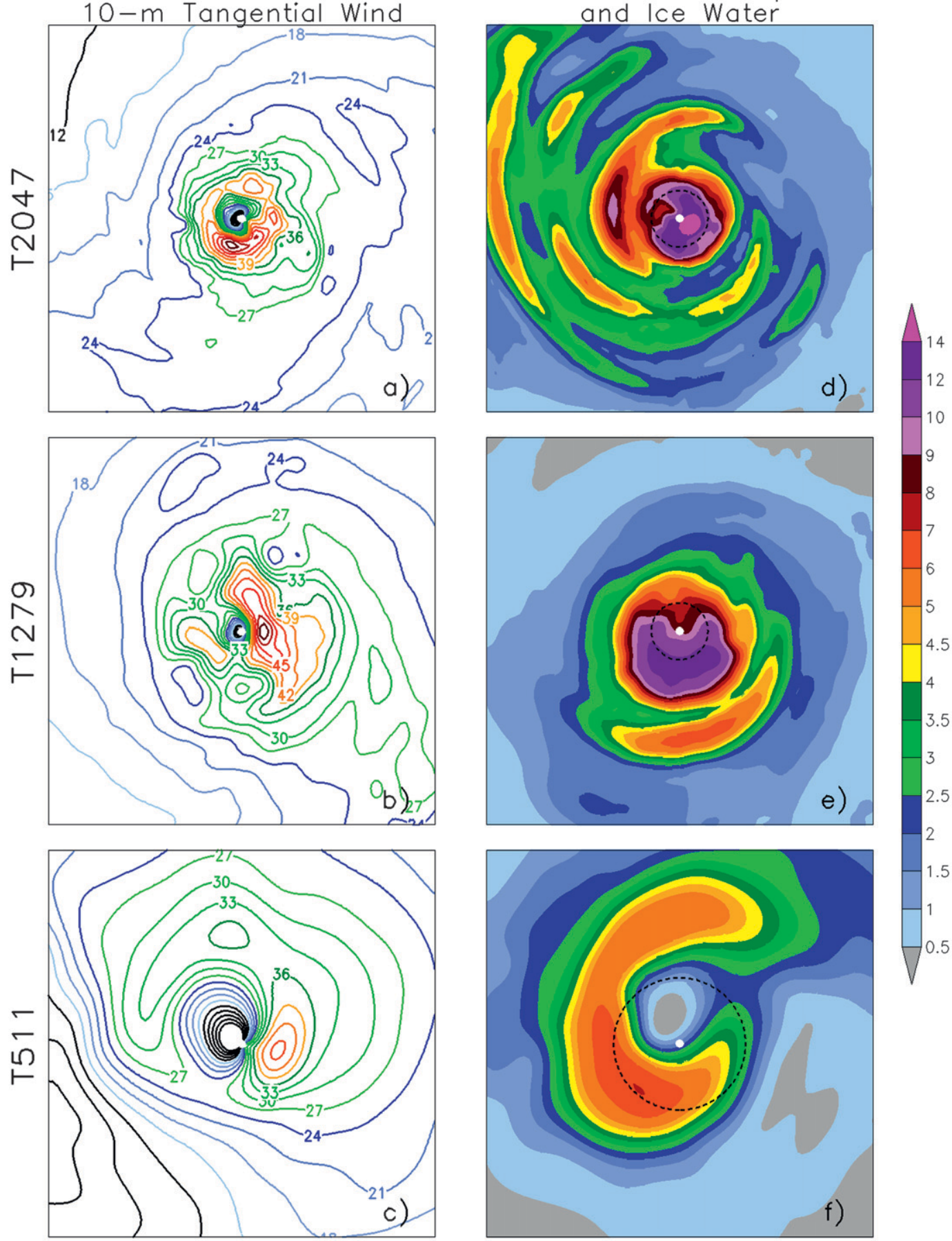

FIG. 10. (left) 10-m tangential wind and (right) TCLIW for some of the most intense TCs at the peak of their intensity from the (a),(d) IFS T2047; (b),(e) T1279; and (c),(f) T511 simulations. Radius is $2^{\circ}$ from the storm center. Wind units are $\mathrm{m} \mathrm{s}^{-1}$. TCLIW units are $\mathrm{kg} \mathrm{m}^{-2}$. Contour interval is $3 \mathrm{~m} \mathrm{~s}^{-1}$. Dashed black contours on the right show RMW for each case.

RMW is $69 \mathrm{~km}$, which somewhat exceeds the 20-60-km range characteristic of TCs. Many lower-resolution models have difficulty simulating the eye, eyewall, and spiral rainbands. At T511, the eye is large compared to observations (see, e.g., Weatherford and Gray 1988b), the eyewall is rather blurred, and the rainbands are not discernible (Fig. 10f). At the higher resolutions, particularly at T2047 for the cases presented, these features are better 


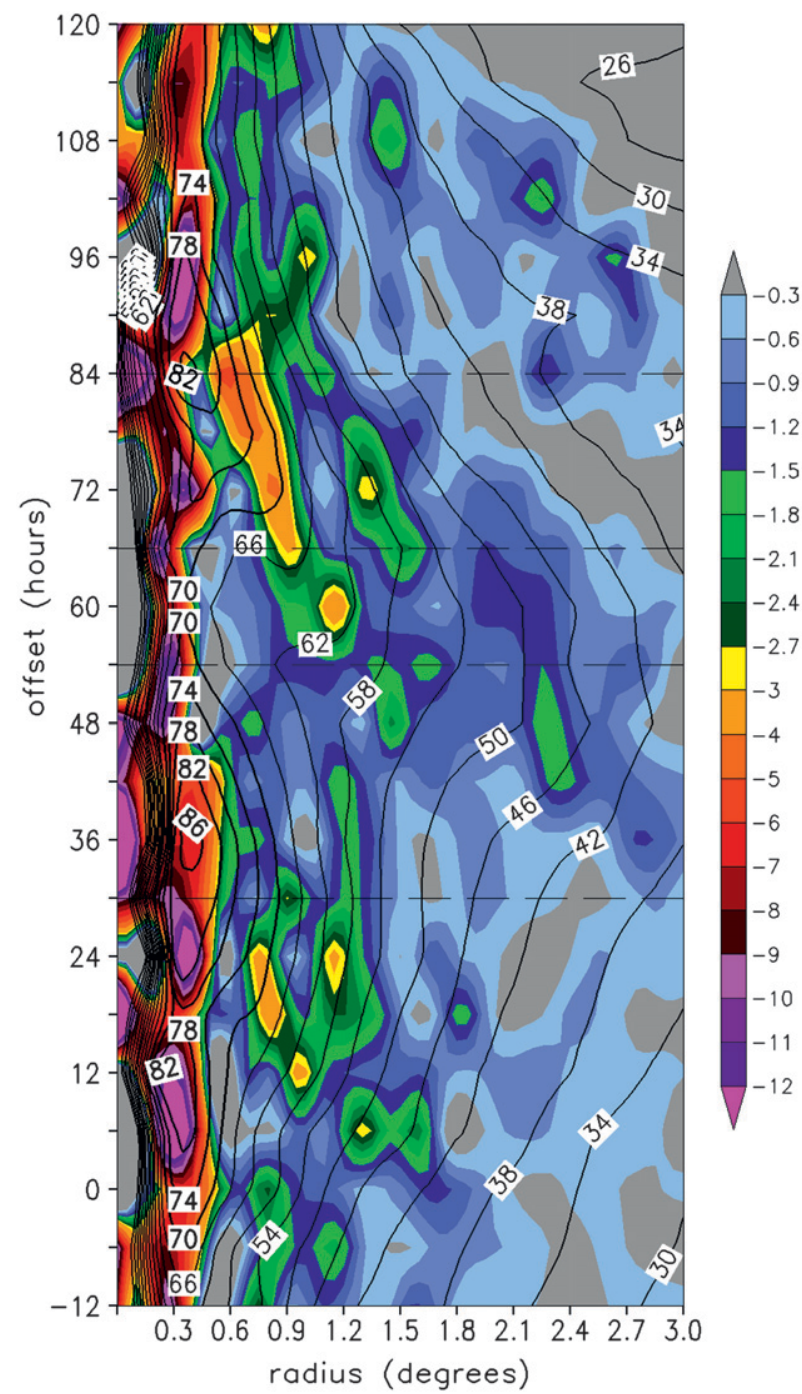

FIG. 11. Radius-time Hovmöller diagram of the azimuthal mean tangential velocity at $850 \mathrm{hPa}$ (contour, $\mathrm{m} \mathrm{s}^{-1}$ ) and omega at $700 \mathrm{hPa}$ (shading, $\mathrm{Pa} \mathrm{s}^{-1}$ ) for an intense TC in the T2047 simulation. Contour interval is $4 \mathrm{~m} \mathrm{~s}^{-1}$.

represented. The model is capable of generating an eye of quite realistic size (Figs. 10a,d), although at 10-km grid spacing it is barely resolved. There is a tight eyewall just outside the RMW (shown by dashed black contours in Figs. 10d-f) including multiple spiral rainbands at varying radii from the storm center (Fig. 10d). In the T1279 case, the eye is less visible, the gradients in the eyewall are weaker, and there is only one rainband present (Fig. 10e).

\section{c. Life cycle of an intense TC in the 10-km simulation}

In this section, we focus on a fragment of the life cycle of one of the most intense TCs in the T2047 simulation. We intend to demonstrate that this storm undergoes intensity fluctuations that occur in apparent connection with the variations of the eyewall/rainband structure, which in many aspects resemble reality. A concise (azimuthally averaged) view of the TC evolution is shown in Fig. 11, where a portion of the life cycle from $12 \mathrm{~h}$ before to $120 \mathrm{~h}$ after the peak intensity (zero offset hour), based on the surface wind speed, is displayed. ${ }^{4}$ The intensity of the TC, estimated here by the magnitude of the 850-hPa tangential wind, undergoes a number of fluctuations reaching peaks at about 12,36 , and $84 \mathrm{~h}(10-\mathrm{m}$ tangential velocity also displays peaks at the same time intervals, in addition to the zero hour-not shown). Three phases of the TC evolution can be distinguished. Phase I (30-54 h) is marked by the weakening of the TC from about 86 to $70 \mathrm{~m} \mathrm{~s}^{-1}$, which coincides with the strengthening of convective motions as far as 3 degrees from the storm center and their inward propagation. The storm expands, as seen, for example, from the $42 \mathrm{~m} \mathrm{~s}^{-1}$ isotach moving to larger distances from the storm center. The bending of isotachs suggests the development of the secondary wind maxima collocated with the strongest convective motion. In Phase II (54-66 h), the storm is weak and diffuse. An additional wind maximum is present at about 1.2 degrees coincident with the center of strong convection. Phase III (66-84 h) signifies reintensification of the storm. At this time, the eyewall strengthens and the storm contracts, while the strong convection that developed in Phase II merges with the recovering eyewall.

Compared with much higher-resolution mesoscale model simulations (e.g., Fig. 2 in Zhou and Wang 2009), Fig. 11 shows many similarities, like the weakening of the storm while the new convection emerges outside the eyewall and the subsequent eyewall reformation and the storm reintensification. These processes do not occur as single events, and may be seen between 84-108 and 0-18 h (Fig. 11), which is also characteristic of observed TCs. There are, however, certain differences. Concentric eyewalls are not present at any stage of the life cycle examined, as shown in more detail below. Instead, the secondary eyewall is weaker and fairly asymmetric. Also, the recovered eyewall $(84 \mathrm{~h})$, including its RMW, does not differ much from the original $(36 \mathrm{~h})$. Considering these differences, it is more likely that the coherent evolution of the TC intensity and structure in Fig. 11 is a result of the interaction of the TC with unfavorable conditions, such as an area of high wind shear, followed by a recovery, rather than an attempt by the model to simulate the eyewall replacement cycle (Willoughby et al. 1982;

\footnotetext{
${ }^{4}$ For the analysis presented in this section, all variables were sampled at their native resolution. The T511 resolution was insufficient to resolve small-scale structure in the rainbands and particularly the inner core of the TC.
} 
Houze et al. 2007). On its path starting at about $30 \mathrm{~h}$, this TC encounters areas of high wind shear in the vicinity of the Philippines (not shown) that are located to the east and south with respect to its direction of motion.

Figures 12 and 13 show a two-dimensional view of the three phases. At the beginning of phase I, the cyclone has a tight and fairly symmetric inner core (except for the wave-2 asymmetry in the eyewall-Fig. 12a), with two major outer rainbands at about 2.0-2.5 degrees to the west and the east (Fig. 12c). These rainbands contain large areas of upward motion (Fig. 12b). In the next six hours, a secondary horizontal wind maximum (SHWM; Samsury and Zipser 1995) emerges in the southwestern quadrant (Fig. 12d; blue shading roughly delineates the regions where the local wind maxima occur). It is associated with the strengthened outer rainband (Figs. 12e,f) that was already present six hours earlier. In the next 12-24 hours, the existing rainband structure amplifies further, leading to multiple sections of convective motion and the associated SHWM that almost encircle the inner core. At some point $(48-60 \mathrm{~h})$, there seem to be two SHWM at different distances across from the storm center and to the south/southeast from it (Figs. 12j,m and Fig. 13a). It is likely that these changes lead to a systematic weakening of the storm's inner core (Figs. 12d,g,j,m and Fig. 13a). Outer rainbands are known to be highly convective (Barnes et al. 1983). SHWM are often associated with rainbands and may develop an "eyewalllike" quality including upward motion in their vicinity (Samsury and Zipser 1995). As a result, large convectively active rainbands with SHWM can act as a partial mesoscale barrier to the inflow into the inner eyewall leading to its gradual erosion (Barnes et al. 1983, 1991; Samsury and Zipser 1995). This scenario is probably exaggerated in the current case since the simulated convective motion in the rainband is rather uniform and sometimes comparable in magnitude to the convection in the eyewall, whereas in reality it is weaker and has more of a cellular and smallscale structure (e.g., Barnes et al. 1983, 1991). This apparent discrepancy could be attributed to the $10-\mathrm{km}$ resolution, which is too coarse to accurately represent rainband structure. We also note that the distribution of vertical motion (hydrostatic pressure velocity) inside the eye is not always realistic either; although substantial downward motion in the eye is present at times (not shown), it is not always collocated with the storm center or surrounded by the ring of deep convection. Most likely, model resolution of $10 \mathrm{~km}$ is insufficient as well to resolve sharp gradients in vertical motion between the eyewall and the eye (within 0.3 degrees of the storm center).

At the beginning of phase III (66 h), qualitatively different processes start taking place. At this time, major rainbands in the southwestern quadrant (Figs. 13b,c) merge (Figs. 13h,i) and start spiraling cyclonically inward toward the eyewall. This is followed by the intensification and contraction of the inner core (Figs. 13j, 1,m,o). The described processes are reminiscent of the scenario when the eyewall recovery and the TC reintensification occur as a result of an inward-propagating outer rainband and its axisymmetrization (May and Holland 1999; Fudeyasu et al. 2010).

\section{Interannual variability of the TC activity}

The capability of a model to reproduce the observed TC variability is an important component in assessing its overall performance in simulating TC statistics. It is also another tough test to pass to be considered for climate change projections. To reduce the stochastic component in the modeled TC variability, an ensemble approach is highly recommended (e.g., Vitart et al. 1997). Since Project Athena did not generate ensemble simulations, we are limited in performing this analysis, as briefly mentioned in the introduction. Below, we present a limited set of diagnostics to shed some light on this aspect of the simulations.

Table 4 shows the skill of the model in reproducing yearto-year variations of the seasonal TC frequency measured by linear correlation with the observed TC frequency for each individual model and a multimodel ensemble mean. The latter is formed to reduce uncertainly and is a measure of the skill of the IFS independent of the resolution, although four members may be insufficient. The correlation of the ensemble-mean and observed TC frequencies is not particularly high but positive and significant for each basin examined, except for the north Indian Ocean. Correlations appear to be higher for the two highest-resolution models, but the spread between the individual values is quite large. Detrending of the time series does not largely change the results.

The El Niño-Southern Oscillation (ENSO) is known to be a strong driver of the interannual variability of the TC frequency in the North Atlantic (Gray 1984) and to a lesser extent in the northeastern and the northwestern Pacific (see review in Camargo et al. 2007a, also Wang and Lee 2009). The correlation of the multimodel ensemble mean TC frequency with the Niño-3.4 index is excellent for the North Atlantic and the northwest Pacific and somewhat weaker for the northeast Pacific (Table 5). Correlations are higher for the two highestresolution runs in two out of three basins. There is again a large spread in correlations among the individual models.

Since ENSO is also known to influence other measures of the TC activity, like the intensity and lifetime (e.g., Camargo et al. 2007a), it is worth examining its 

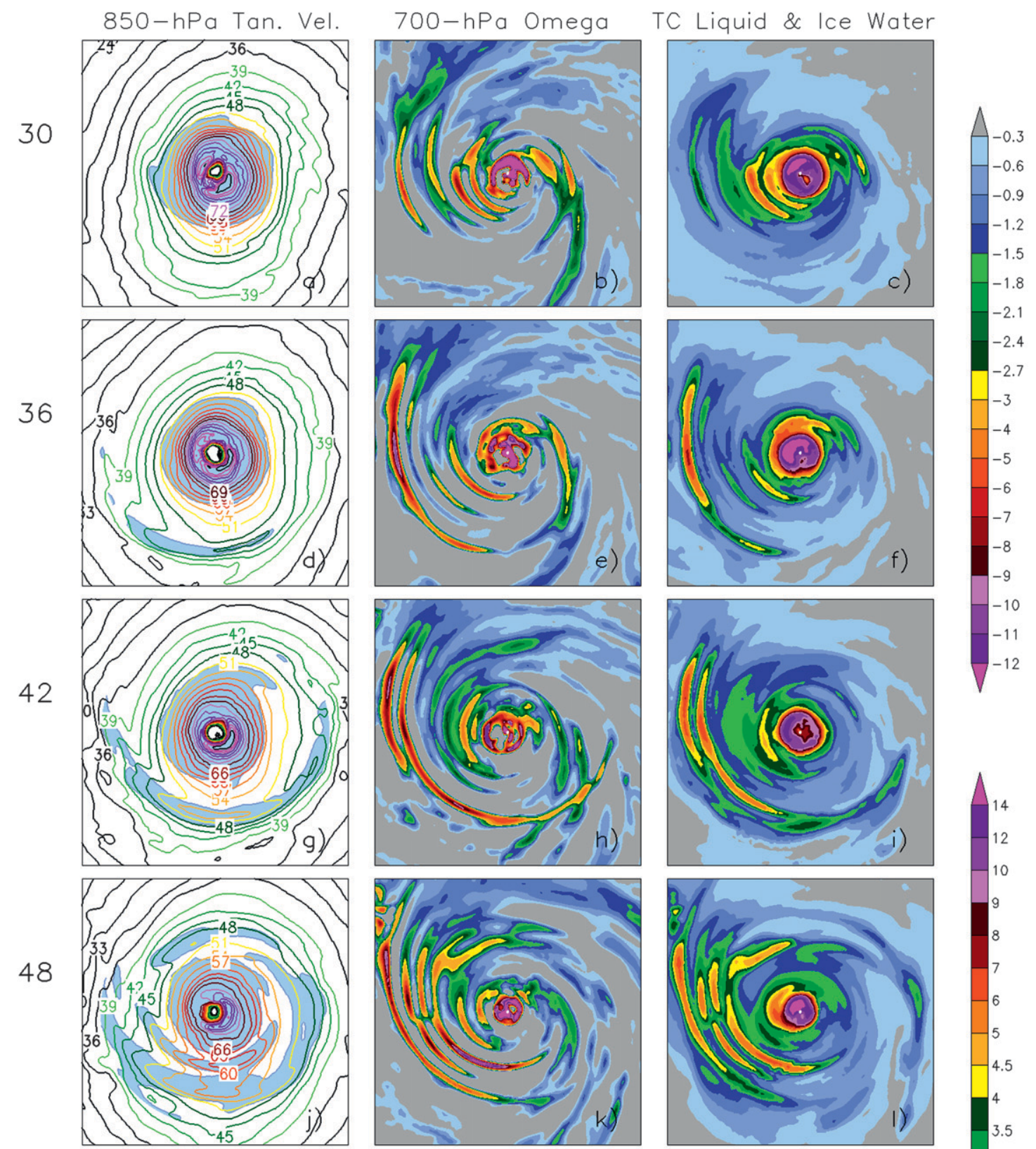

48
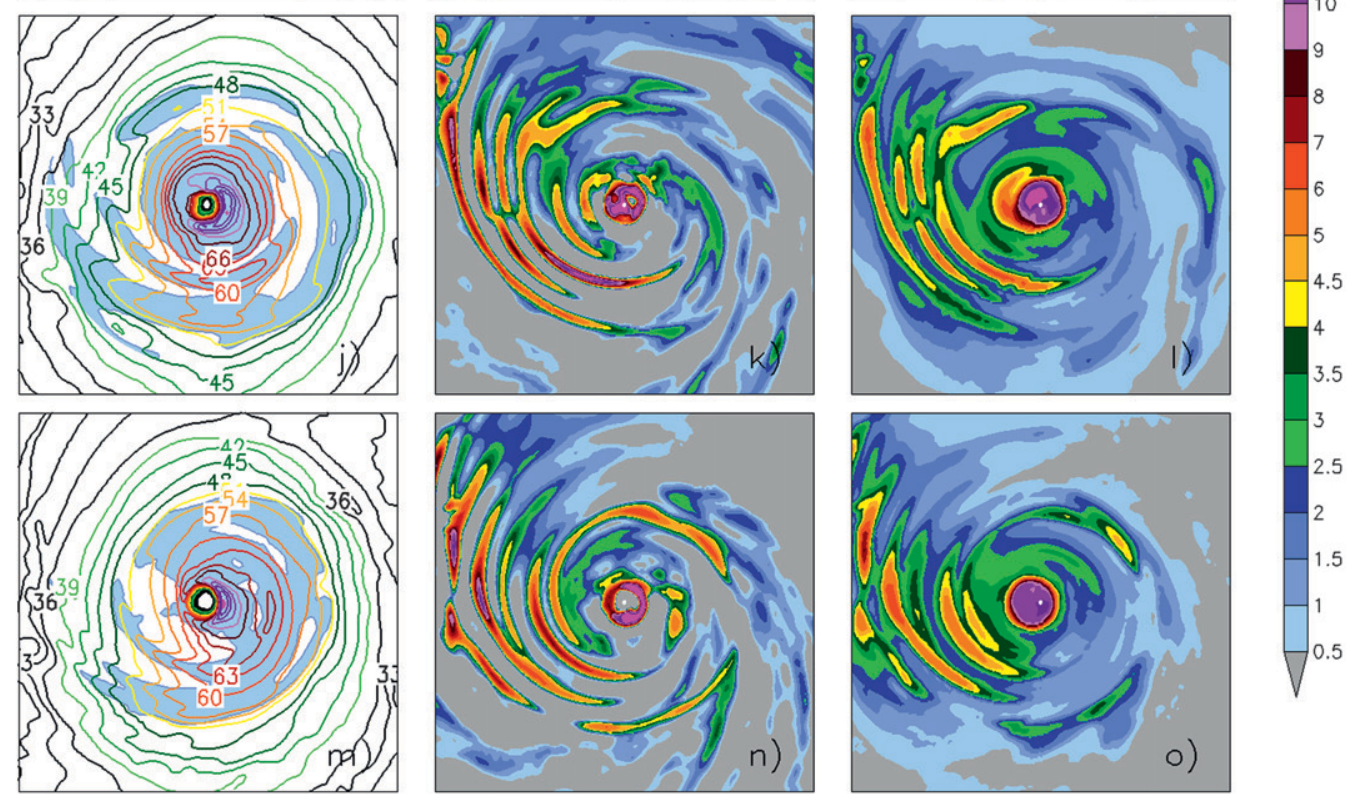

FIG. 12. (left) 850-hPa tangential velocity, (middle) 700-hPa omega, and (right) TCLIW for the offset hours of 30-54 shown on the left for an intense TC in the T2047 simulation. Radius is $3.5^{\circ}$ from the storm center. Velocity units are $\mathrm{m} \mathrm{s}^{-1}$. Omega units are $\mathrm{Pa} \mathrm{s}^{-1}$. TCLIW units are $\mathrm{kg} \mathrm{m}^{-2}$. Contour interval is $3 \mathrm{~m} \mathrm{~s}^{-1}$. Omega (TCLIW) shading interval is shown by a color bar in the upper (lower) right. Blue shading on the left roughly delineates the regions where the local wind maxima occur. 

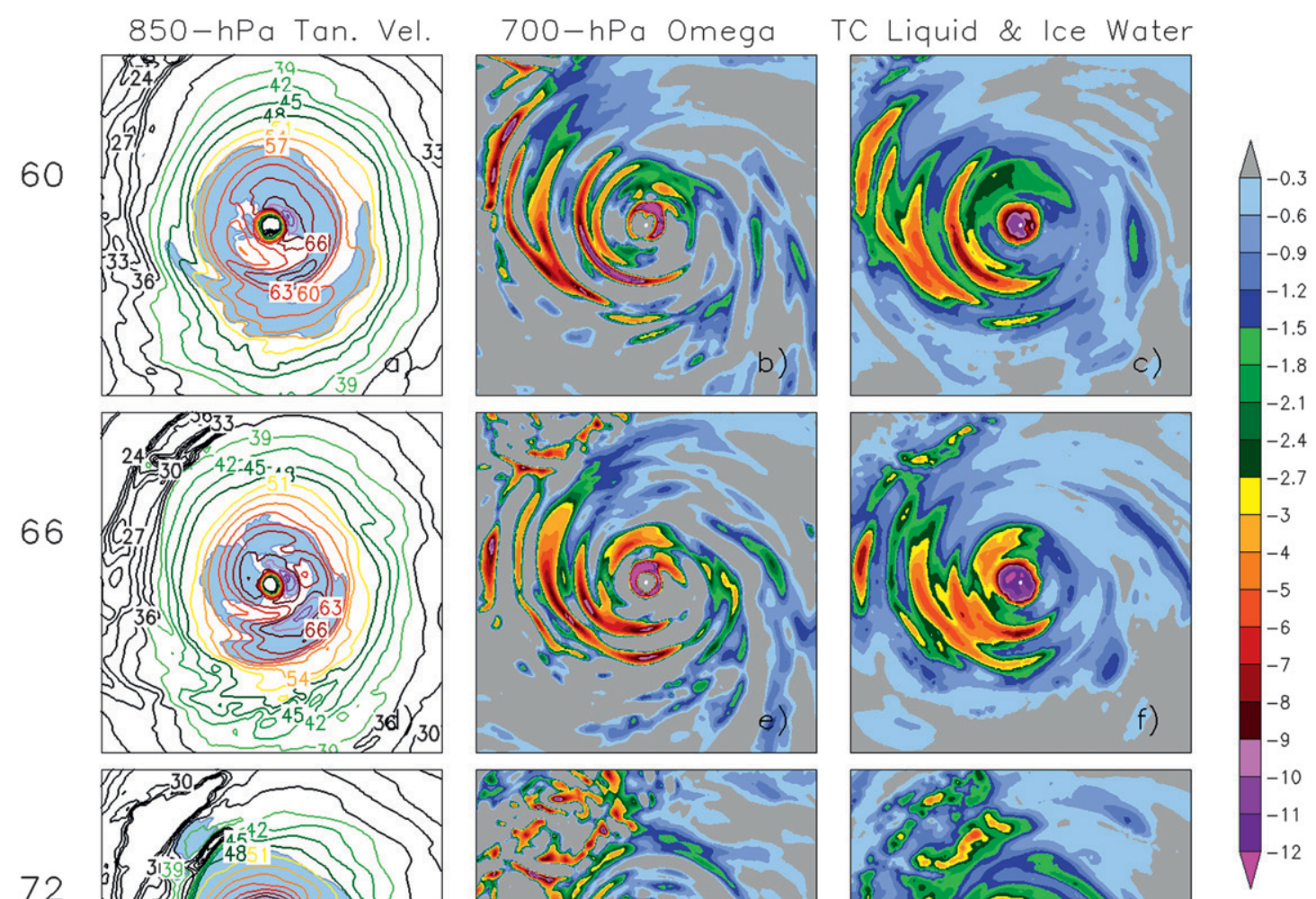

72
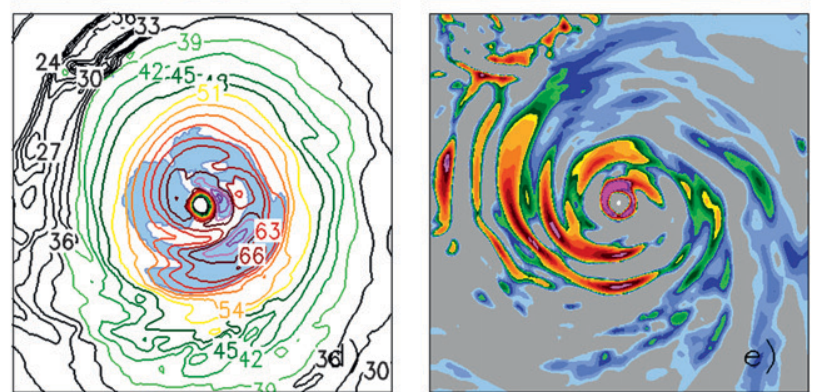

78
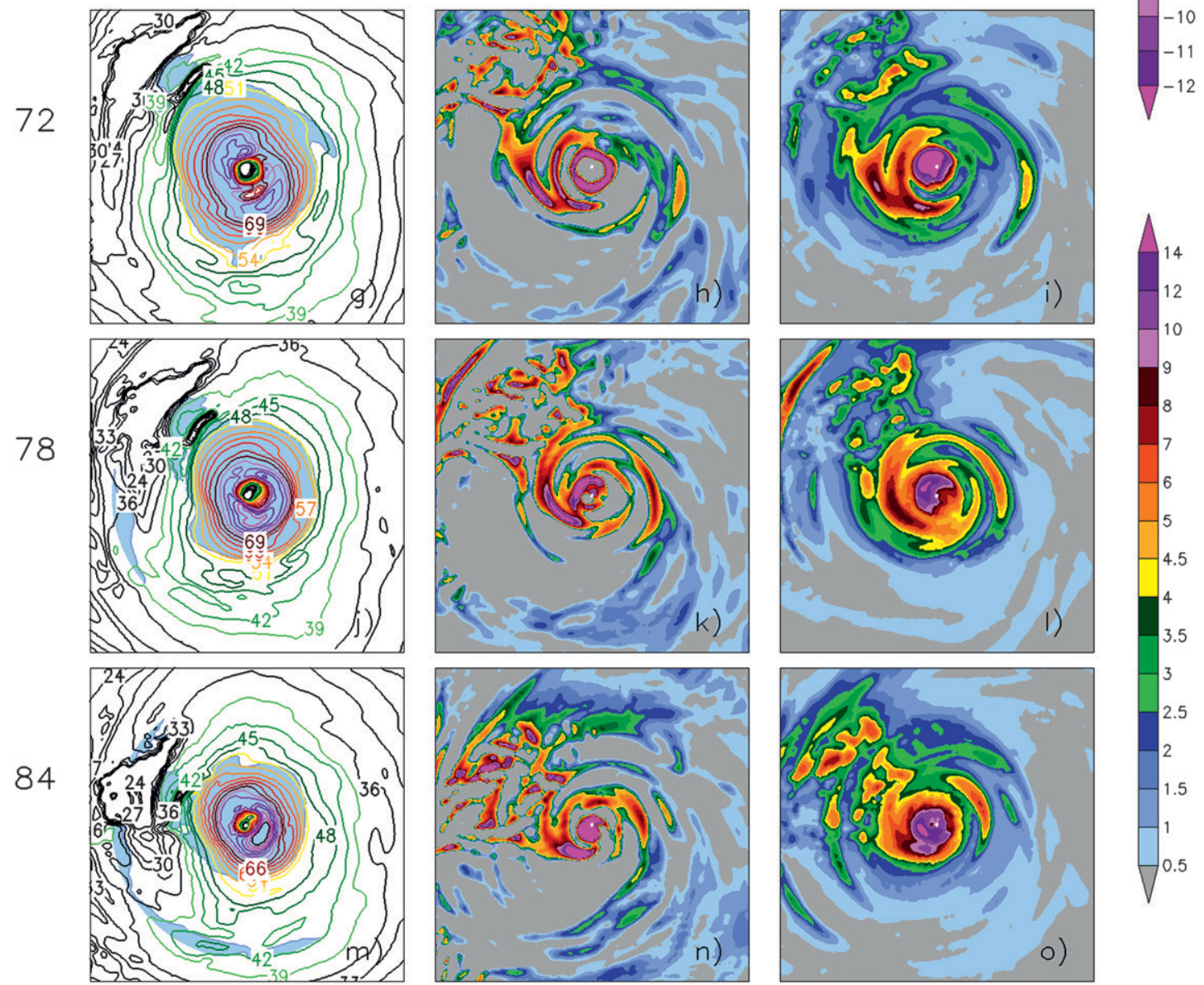

84
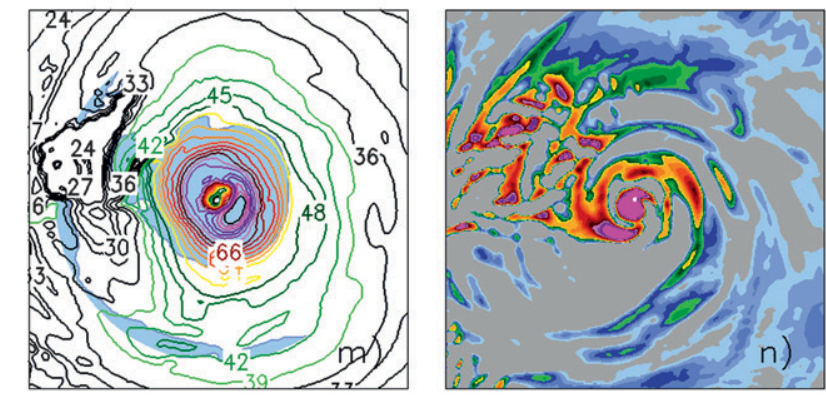

FIG. 13. As in Fig. 12, but for the offset hours of 60-84 shown on the left. 
TABLE 4. Correlation coefficients between the MJJASON-average simulated and observed (IBTrACS) TC frequency during 19902008. (Correlation coefficients for the detrended time series are given in the parenthesis.) Bold values indicate that the correlation coefficient is statistically significant at the $95 \%$ confidence level using a one-sided Student's $t$ test and assuming temporal independence of each record.

\begin{tabular}{lccrrr}
\hline \hline & Ensemble mean* & T2047 & T1279 & T511 & T159 \\
\hline North Atlantic & $\mathbf{0 . 4 8}(\mathbf{0 . 4 6})$ & $0.36(\mathbf{0 . 4 0})$ & $\mathbf{0 . 5 8}(\mathbf{0 . 5 2})$ & $0.14(0.17)$ & $0.32(0.26)$ \\
Northeast Pacific & $\mathbf{0 . 4 7}(0.37)$ & $0.37(0.24)$ & $\mathbf{0 . 6 1}(\mathbf{0 . 5 4})$ & $\mathbf{0 . 3 9}(0.34)$ & $0.26(0.12)$ \\
Northwest Pacific & $\mathbf{0 . 5 8}(\mathbf{0 . 5 2})$ & $\mathbf{0 . 5 4}(\mathbf{0 . 4 4})$ & $\mathbf{0 . 4 4}(0.28)$ & $0.27(0.33)$ & $0.29(0.30)$ \\
North Indian & $-0.04(-0.04)$ & $0.06(0.04)$ & $-0.25(-0.32)$ & $-0.18(-0.11)$ & $0.13(0.11)$ \\
\hline
\end{tabular}

* The ensemble mean is computed by averaging all four experiments after calibration, which is done by multiplying each time series by a constant factor so that the mean TC frequency over 1990-2008 for each resolution is equal to the observed.

effect on the power dissipation index (PDI), which is an integral measure of the TC activity (Emanuel 2005). The correlation of the multimodel ensemble mean PDI with the Niño-3.4 index is very good, except in the North Atlantic where it is practically zero (Table 6). The ensemble mean PDI has also a high correlation with the observed PDI in the northeastern and northwestern Pacific, whereas it is low and insignificant in the North Atlantic (not shown). The apparent failure of the IFS to simulate interannual variability of this quantity in the North Atlantic is unclear and currently under investigation.

\section{Summary and conclusions}

As computing power continues to grow, it is becoming increasingly possible to model the global climate at horizontal resolutions presently used in short-term weather prediction. As part of Project Athena, a unique set of multiyear global climate simulations with the ECMWF IFS at the following resolutions has been produced: $125 \mathrm{~km}$ (representative of current climate models), $39 \mathrm{~km}$ (generally found in regional climate models), $16 \mathrm{~km}$ (currently used for medium-range weather prediction), and $10 \mathrm{~km}$ (used in mesoscale prediction models). In this paper, a comparative analysis of the TC climatology based on the above dataset is presented.

We note that our TC identification method is heavily based on the surface $(10-\mathrm{m})$ wind speed threshold, adjusted for model resolution as per Walsh et al. (2007) and model time step where applicable. The structural (warm core) criteria are very general, requiring only that the vorticity maxima exist at each level, and the vorticity difference between the lower and upper troposphere is larger than zero (Table 1). While our goal is to use conditions that would allow objective comparison with the observations and between different model versions (i.e., resolutions), it must be acknowledged that this set of choices in itself represents a stringent test on the model performance, especially in terms of getting the TC count right.

The spatial distributions of genesis and tracks in the North Pacific do not exhibit much sensitivity to the model resolution higher than $39 \mathrm{~km}$. At the lowest two resolutions, there are some discrepancies as follows: regional shifts in the centers of cyclogenesis in the northwest Pacific and lower activity in the South China Sea and particularly the northeast Pacific at T159. Apart from these differences, the IFS generally produces too many TCs in the northwest Pacific and too few in the northeast Pacific, although the mean TC frequency in the latter basin in the 10- and 16-km models is not significantly different from that observed. Analysis of the large-scale conditions suggests that these errors could be related to the quality of the model precipitation over the western Pacific warm pool and the ITCZ, the mean bias of which is rather similar among the four resolutions studied. In the North Atlantic on the other hand, the distribution of genesis and track densities shows systematic improvement with the resolution increase, although the total storm count remains low. The $10-\mathrm{km}$, and to a lesser

TABLE 5. Correlation coefficients between the MJJASON average TC frequency and the Niño-3.4 index during 1990-2007. Bold values indicate that the correlation coefficient is statistically significant at the $95 \%$ confidence level using a one-sided Student's $t$ test and assuming temporal independence of each record.

\begin{tabular}{|c|c|c|c|c|c|c|}
\hline & Obs (IBTrACS) & Ensemble mean* & T2047 & T1279 & T511 & $\mathrm{T} 159$ \\
\hline North Atlantic & -0.50 & -0.55 & -0.63 & -0.30 & -0.17 & -0.45 \\
\hline Northeast Pacific & 0.44 & 0.27 & 0.28 & 0.32 & 0.27 & 0.08 \\
\hline Northwest Pacific & 0.46 & 0.42 & 0.38 & 0.45 & -0.09 & 0.30 \\
\hline
\end{tabular}

\footnotetext{
* Ensemble means are computed as in Table 4.
} 
TABLE 6. Correlation coefficients between the MJJASON average PDI and the Niño-3.4 index during 1990-2007. Bold values indicate that the correlation coefficient is statistically significant at the $95 \%$ confidence level using a one-sided Student's $t$ test and assuming temporal independence of each record.

\begin{tabular}{|c|c|c|c|c|c|c|}
\hline & Obs & $\begin{array}{c}\text { Ensemble } \\
\text { mean* }\end{array}$ & T2047 & T1279 & T511 & $\mathrm{T} 159$ \\
\hline North Atlantic & -0.48 & -0.09 & 0.01 & 0.2 & -0.15 & -0.26 \\
\hline Northeast Pacific & 0.53 & 0.57 & 0.32 & 0.64 & 0.52 & 0.32 \\
\hline Northwest Pacific & 0.74 & 0.68 & 0.67 & 0.64 & 0.33 & 0.35 \\
\hline
\end{tabular}

* Ensemble means are computed as in Table 4.

extent the $16-\mathrm{km}$, models are the only ones in the set that generate a realistic number of North Atlantic TCs. In addition, the T2047 model shows a qualitatively better simulation of the track densities in the subtropical North Atlantic compared to the other model versions: the region of the highest concentration of tracks shifts westward from the center of the basin closer to the east coast of the United States more in line with the observations. This improvement could potentially lead to a more realistic distribution of hurricane landfall.

The main advantages of the model with the $10-\mathrm{km}$ (and 16-km) grid spacing are found to be associated with the quality of the intensity distribution and the threedimensional structure of the modeled storms, which show dramatic improvement compared to the two lower resolutions as now detailed.

1) The full distribution of the TC intensity and particularly its tail (maximum surface wind speeds of $35 \mathrm{~m} \mathrm{~s}^{-1}$ or higher, or minimum SLP of $960 \mathrm{hPa}$ or lower) attain realistic shape only at T1279 and moreso at T2047. These results suggest that a hydrostatic model with convective parameterization could be efficiently used to simulate the TC intensity response under the climate change scenario if its resolution is increased to about $10 \mathrm{~km}$, provided that the variability of the TC activity in the model is adequately simulated as well. This is a subject of our future research.

2) At the 16- and 10-km resolutions, the model is capable of generating storms equivalent to the supertyphoon category in the northwest Pacific and category 4 (or category 5 in minimum SLP) in the North Atlantic. The most intense TC attains a lifetime maximum surface wind speed or a lifetime minimum SLP of 68.4 (65.2) $\mathrm{m} \mathrm{s}^{-1}$ and 857 (852) hPa at T2047 (T1279) compared to only $46.4 \mathrm{~m} \mathrm{~s}^{-1}$ and $903 \mathrm{hPa}$ at T511. These results generally support the conclusion of Fierro et al. (2009) that a grid spacing of $10 \mathrm{~km}$ is a "threshold" at which the TC intensity is expected to increase dramatically. One reason for this is that it represents a lower limit of the horizontal resolution at which a typical eyewall can be resolved. However, in our experiments intensities in the 10- and $16-\mathrm{km}$ integrations are fairly similar suggesting that the exact threshold may be somewhat model-dependent. The actual values of the peak intensities given above need to be taken with caution, however, as they are likely exaggerated because of the absence of an interactive ocean. A coupled modeling study at the highest resolutions would be useful to clarify these influences.

3) We find a significant difference in the simulation of the three-dimensional structure of the most intense storms even in the comparison of the 39- to the $16-\mathrm{km}$ resolution, which is not directly related to the intensity differences of the TCs studied. (Results from the 16- and 10-km models are fairly similar.) The higher-resolution storms have on average more intense warm cores of quite realistic magnitudes, higher wind maxima, smaller and also more realistic RMWs, and consequently stronger radial temperature and velocity gradients in the eyewall. The surface structure shows significantly more complexity with smaller scale and greater detail in the inner core, the presence of an eye surrounded by a tight eyewall, and multiple spiral rainbands. This is in stark contrast with the T511 simulation where these features are either diffuse or absent altogether. In addition, our TC life cycle analysis shows that in the T2047 model, TC intensity fluctuations occur in conjunction with the structural changes that appear to mimic reality. These results are again rather encouraging and indicate that in a climate change simulation at these highest resolutions, it would be possible not only to adequately assess the TC intensity response but also to take a more detailed look at the structural changes of the storms in their relation to the intensity change.

4) While a nonhydrostatic model that simulates explicitly cloud processes and convection could represent TC features with higher fidelity, it is currently rather costly to run such models (e.g., Kinter et al. 2011, manuscript submitted to Bull. Amer. Meteor. Soc.). Therefore, the use of a high-resolution AGCM with conventional parameterizations could be an intermediate step toward achieving the goal of a more reliable prediction of the $\mathrm{TC}$ response to climate change.

In the future work, we intend to compare the change in the TC statistics in response to a changing climate and its sensitivity to the model resolution using the 16- and $125-\mathrm{km}$ versions of IFS. We also hope to assess the robustness of the results presented here and from the climate change study by comparing with similar results from simulations of the Nonhydrostatic Icosahedral 
Atmospheric Model (NICAM) at the resolution similar to IFS T2047 also conducted under Project Athena.

Acknowledgments. The results described herein were obtained in the 2009/10 Athena Project, a computationally intensive project that was carried out using the Athena supercomputer at the University of Tennessee's National Institute for Computational Sciences (NICS), under the auspices of the National Science Foundation (NSF). Support provided by the NICS, the NSF (Grants 0830068 and 0957884), NOAA (Grant NA09OAR4310058), and NASA (Grant NNX09AN50G) is gratefully acknowledged. We also acknowledge the support of the European Centre for Medium-Range Weather Forecasts (ECMWF), which provided the IFS code, boundary and initial conditions datasets, and run scripts. We are thankful to Dr. David Straus (COLA) for the help with spectral processing and Dr. Xiasong Yang (COLA) for assistance with computing. The following individuals contributed to the project: P. Dirmeyer, B. Doty, and T. Wakefield (COLA); P. Bechtold, M. Fuentes, M. Hamrud, M. Miller, and T. Palmer (ECMWF); M. Satoh (University of Tokyo; Japan); H. Tomita [RIKEN Advanced Institute for Computational Sciences (AICS); Japan]; C. Kodama and Y. Yamada [Japan Agency for Marine-Earth Science and Technology (JAMSTEC); Japan]; P. Andrews, T. Baer, M. Ezell, C. Halloy, D. John, B. Loftis, and K. Wong (NICS; United States); and P. Johnsen and P. Nyberg (Cray, Inc.).

\section{REFERENCES}

Barnes, G. M., E. J. Zipser, D. Jorgensen, and F. Marks, 1983 Mesoscale and convective structure of a hurricane rainband. J. Atmos. Sci., 40, 2125-2137.

_ J. J. Famache, M. A. LeMone, and G. J. Stossmeister, 1991: A convective cell in a hurricane rainband. Mon. Wea. Rev. 119, 776-794.

Bechtold, P., M. Koehler, T. Jung, F. Doblas-Reyes, M. Leutbecher, M. J. Rodwell, F. Vitart, and G. Balsamo, 2008: Advances in simulating atmospheric variability with the ECMWF model: From synoptic to decadal time-scales. Quart. J. Roy. Meteor. Soc., 134, 1337-1351.

Bell, K., and P. Sawin Ray, 2004: North Atlantic hurricanes $1977-$ 99: Surface hurricane force wind radii. Mon. Wea. Rev., 132, 1167-1189.

Bender, M. A., T. R. Knutson, R. E. Tuleya, J. J. Sirutis, G. A. Vecchi, S. T. Garner, and I. M. Held, 2010: Modeled impact of anthropogenic warming on the frequency of intense Atlantic hurricanes. Science, 327, 454-458.

Bengtsson, L., M. Botzet, and M. Esch, 1995: Hurricane-type vortices in a general circulation model. Tellus, 47A, 175-196.

— K. K. Hodges, and M. Esch, 2007a: Tropical cyclones in a T159 resolution global climate model: Comparison with observations and re-analyses. Tellus, 59A, 396-416.

,,--- N. Keenlyside, L. Kornblueh, J.-J. Luo, and T. Yamagata, 2007b: How may tropical cyclones change in a warmer climate? Tellus, 59A, 539-561.
Camargo, S. J., A. G. Barnston, and S. E. Zebiak, 2005: A statistical assessment of tropical cyclone activity in atmospheric general circulation models. Tellus, 57A, 589-604.

$\longrightarrow$, K. A. Emanuel, and A. H. Sobel, 2007a: Use of a genesis potential index to diagnose ENSO effects on tropical cyclone genesis. J. Climate, 20, 4819-4834.

- A. H. Sobel, A. G. Barnston, and K. A. Emanuel, 2007b: Tropical cyclone genesis potential index in climate models. Tellus, 59A, 428-443.

Caron, L.-P., J. G. Colin, and K. Winger, 2010: Impact of resolution and downscaling technique in simulating recent Atlantic tropical cyclone activity. Climate Dyn., 37, 869-892, doi:10.1007/ s00382-010-0846-7.

Catto, J. L., L. C. Shaffrey, and K. I. Hodges, 2010: Can climate models capture the structure of extratropical cyclones? J. Climate, 23, 1621-1635.

Chen, S. S., J. F. Price, W. Zhao, M. A. Donelan, E. J. Walsh, and H. L. Tolman, 2007: The CBLAST hurricane program and the next-generation fully coupled atmosphere-wave-ocean models for hurricane research and prediction. Bull. Amer. Meteor. Soc., 88, 311-317.

Dirmeyer, P. A., and Coauthors, 2012: Simulating the diurnal cycle of rainfall in global climate models: Resolution versus parameterization. Climate Dyn., doi:10.1007/s00382-011-1127-9, in press.

Emanuel, K., 2003: Tropical cyclones. Annu. Rev. Earth Planet. Sci., 31, 75-104.

- 2005: Increasing destructiveness of tropical cyclones over the past 30 years. Nature, 436, 686-688, doi:10.1038/nature03906.

European Centre for Medium-Range Weather Forecasts, cited 2009: IFS documentation-Cy33r1: Operational implementation 3 June 2008. [Available online at http://www.ecmwf.int/research/ ifsdocs/CY33r1/index.html.]

Fierro, A. O., R. F. Rogers, F. D. Marks, and D. S. Nolan, 2009: The impact of horizontal grid spacing on the microphysical and kinematic structures of strong tropical cyclones simulated with the WRF-ARW model. Mon. Wea. Rev., 137, 3717-3743.

Frank, W. M., 1977: The structure and energetics of the tropical cyclone. Part I: Storm structure. Mon. Wea. Rev., 105, 1119-1135.

Fudeyasu, H., Y. Wang, M. Satoh, T. Nasuno, H. Miura, and W. Yanase, 2010: Multiscale interactions in the life cycle of a tropical cyclone simulated in a global cloud system-resolving model. Part I: Large-scale and storm-scale evolutions. Mon. Wea. Rev., 138, 4285-4304.

Gentry, M. S., and G. M. Lackmann, 2010: Sensitivity of simulated tropical cyclone structure and intensity to horizontal resolution. Mon. Wea. Rev., 138, 688-704.

Gopalakrishnan, S. G., F. Marks, X. Zhang, J.-W. Bao, K.-S. Yeh, and R. Atlas, 2011: The experimental HWRF system: A study on the influence of horizontal resolution on the structure and intensity changes in tropical cyclones using an idealized framework. Mon. Wea. Rev., 139, 1762-1784.

Gray, W. M., 1984: Atlantic seasonal hurricane frequency. Part I: El Niño and 30-mb quasi-biennial oscillation influences. Mon. Wea. Rev., 112, 1649-1668.

Harper, B. A., J. D. Kepert, and J. D. Ginger, 2009: Guidelines for converting between various wind averaging periods in tropical cyclone conditions. World Meteorological Organization Rep. TCM-VI/Doc. 2.3, 62 pp.

Hodges, K. I., 1995: Feature tracking on the unit sphere. Mon. Wea. Rev., 123, 3458-3465.

1996: Spherical nonparametric estimators applied to the UGAMP model integration for AMIP. Mon. Wea. Rev., 124, 2914-2932. 
_ 1999: Adaptive constraints for feature tracking. Mon. Wea. Rev., 127, 1362-1373.

Houze, R. A., S. S. Chen, B. F. Smull, W.-C. Lee, and M. M. Bell, 2007: Hurricane intensity and eyewall replacement. Science, 315, 1235-1239.

Jung, T., and Coauthors, 2010: The ECMWF model climate: Recent progress through improved physical parametrizations. Quart. J. Roy. Meteor. Soc., 136, 1145-1160.

_ , and Coauthors, 2012: High-resolution global climate simulations with the ECMWF model in Project Athena: Experimental design, model climate, and seasonal forecast skill. J. Climate, 25, 31553172.

Kepert, J. D., 2006a: Observed boundary layer wind structure and balance in the hurricane core. Part I: Hurricane Georges. J. Atmos. Sci., 63, 2169-2193.

_ 2006b: Observed boundary layer wind structure and balance in the hurricane core. Part II: Hurricane Mitch. J. Atmos. Sci., 63, 2194-2211.

Kimball, S. K., and M. S. Mulekar, 2004: A 15-yr climatology of North Atlantic tropical cyclones. Part I: Size parameters. J. Climate, 17, 3555-3575.

Knaff, J. A., and R. M. Zehr, 2007: Reexamination of tropical cyclone wind-pressure relationships. Wea. Forecasting, 22, 71-88.

Knapp, K. R., M. C. Kruk, D. H. Levinson, H. J. Diamond, and C. J. Neumann, 2010: The International Best Track Archive for Climate Stewardship (IBTrACS). Bull. Amer. Meteor. Soc., 91, 363-376.

Knutson, T. R., J. J. Sirutis, S. T. Garner, I. M. Held, and R. E. Tuleya, 2007: Simulation of the recent multidecadal increase of Atlantic hurricane activity using an $18-\mathrm{km}$-grid regional model. Bull. Amer. Meteor. Soc., 88, 1549-1565.

Köhler, M., 2005: Improved prediction of boundary layer clouds. ECMWF Newsletter, No. 104, ECMWF, Reading, United Kingdom, 18-22.

LaRow, T. E., Y.-K. Lim, D. W. Shin, E. P. Chassignet, and S. Cocke, 2008: Atlantic basin seasonal hurricane simulations. J. Climate, 21, 3191-3206.

Lin, I.-I., C.-C. Wu, I.-F. Pun, and D.-S. Ko, 2008: Upper-ocean thermal structure and the western North Pacific category 5 typhoons. Part I: Ocean features and the category 5 typhoons' intensification. Mon. Wea. Rev., 136, 3288-3306.

Liou, C.-S., 2007: Sensitivity of high-resolution tropical cyclone intensity forecasts to surface flux parameterization. Nat. Hazards, 41, 387-399.

Mallen, K. J., M. T. Montgomery, and B. Wang, 2005: Reexamining the near-core radial structure of the tropical cyclone primary circulation: Implications for vortex resiliency. J. Atmos. Sci., 62, 408-425.

May, P. T., and G. J. Holland, 1999: The role of potential vorticity generation in tropical cyclone rainbands. J. Atmos. Sci., 56, 1224-1228.

Molinari, J., and D. Vollaro, 2000: Planetary- and synoptic-scale influences on eastern Pacific tropical cyclogenesis. Mon. Wea. Rev., 128, 3296-3307.

Murakami, H., and M. Sugi, 2010: Effect of model resolution on tropical cyclone climate projections. SOLA, 6, 73-76.

Oouchi, K., J. Yoshimura, H. Yoshimura, R. Mizuta, S. Kusunoki, and A. Noda, 2006: Tropical cyclone climatology in a global warming climate as simulated in a 20-km mesh global atmospheric model: Frequency and wind intensity analysis. J. Meteor. Soc. Japan, 84, 259-276.

Powell, M. D., P. J. Vickery, and T. A. Reinhold, 2003: Reduced drag coefficient for high wind speeds in tropical cyclones. Nature, 422, 279-283.
Samsury, C. E., and E. J. Zipser, 1995: Secondary wind maxima in hurricanes: Airflow and relationship to rainbands. Mon. Wea. Rev., 123, 3502-3517.

Serra, Y. L., G. N. Kiladis, and K. I. Hodges, 2010: Tracking and mean structure of easterly waves over the intra-Americas sea. J. Climate, 23, 4823-4840.

Shen, B.-W., R. Atlas, O. Oreale, S.-J. Lin, J.-D. Chern, J. Chang, C. Henze, and J.-L. Li, 2006: Hurricane forecasts with a global mesoscale-resolving model: Preliminary results with Hurricane Katrina (2005). Geophys. Res. Lett., 33, L13813, doi:10.1029/ 2006GL026143.

Shukla, J., R. Hagedorn, M. Miller, T. N. Palmer, B. Hoskins, J. Kinter, J. Marotzke, and J. Slingo, 2009: Strategies: Revolution in climate prediction is both necessary and possible: A declaration at the World Modelling Summit for Climate Prediction. Bull. Amer. Meteor. Soc., 90, 175-178.

Solomon, S., D. Qin, M. Manning, Z. Chen, M. Marquis, K. B. Averyt, M. Tignor, and H. L. Miller, Eds., 2007: IPCC 2007: The Physical Science Basis. Cambridge University Press, 996 pp.

Stern, D. P., and D. S. Nolan, 2009: Reexamining the vertical structure of tangential winds in tropical cyclones: Observations and theory. J. Atmos. Sci., 66, 3579-3600.

Sundqvist, H., 1978: A parameterization scheme for non-convective condensation including prediction of cloud water content. Quart. J. Roy. Meteor. Soc., 104, 677-690.

Temperton, C., M. Hortal, and A. Simmons, 2001: A two-timelevel semi-Lagrangian global spectral model. Quart. J. Roy. Meteor. Soc., 127, 111-127.

Thorncroft, C., and K. Hodges, 2001: African easterly wave variability and its relationship to Atlantic tropical cyclone activity. J. Climate, 14, 1166-1179.

Tiedtke, M., 1989: A comprehensive mass flux scheme for cumulus parameterization in large-scale models. Mon. Wea. Rev., 117, 1779-1800.

_ 1993: Representation of clouds in large-scale models. Mon Wea. Rev., 121, 3040-3061.

Trenberth, K. E., and J. Fasullo, 2007: Water and energy budgets of hurricanes and implications for climate change. J. Geophys. Res., 112, D23107, doi:10.1029/2006JD008304.

Untch, A., and M. Hortal, 2004: A finite-element scheme for the vertical discretization of the semi-Lagrangian version of the ECMWF forecast model. Quart. J. Roy. Meteor. Soc., 130, 1505-1530.

Uppala, S. M., and Coauthors, 2005: The ERA-40 Re-Analysis. Quart. J. Roy. Meteor. Soc., 131, 2961-3012.

Vitart, F., J. L. Anderson, and W. F. Stern, 1997: Simulation of interannual variability of tropical storm frequency in an ensemble of GCM integrations. J. Climate, 10, 745-760.

Walsh, K. J. E., 2008: The ability of climate models to generate tropical cyclones: Implications for prediction. Climate Change Research Progress, L. Peretz, Ed., Nova Publishers, 313-329.

_ M. Fiorino, C. W. Landsea, and K. L. McInnes, 2007: Objectively determined resolution-dependent threshold criteria for the detection of tropical cyclones in climate models and reanalyses. J. Climate, 20, 2307-2314.

Wang, C., and S.-K. Lee, 2009: Co-variability of tropical cyclones in the North Atlantic and the eastern North Pacific. Geophys. Res. Lett., 36, L24702, doi:10.1029/2009GL041469.

Wang, Y., and C.-C. Wu, 2004: Current understanding of tropical cyclone structure and intensity changes-A review. Meteor. Atmos. Phys., 87, 257-278.

Weatherford, C. L., and W. M. Gray, 1988a: Typhoon structure as revealed by aircraft reconnaissance. Part I: Data analysis and climatology. Mon. Wea. Rev., 116, 1032-1043. 
, and — 1988b: Typhoon structure as revealed by aircraft reconnaissance. Part II: Structural variability. Mon. Wea. Rev., 116, 1044-1056.

Willoughby, H. E., J. A. Clos, and M. G. Shoreibah, 1982: Concentric eye walls, secondary wind maxima, and the evolution of the hurricane vortex. J. Atmos. Sci., 39, 395-411.

Wu, C.-C., C.-Y. Lee, and I.-I. Lin, 2007: The effect of the ocean eddy on tropical cyclone intensity. J. Atmos. Sci., 64, 3562-3578.

Zehnder, J. A., D. M. Powell, and D. L. Ropp, 1999: The interaction of easterly waves, orography, and the intertropical convergence zone in the genesis of eastern Pacific tropical cyclones. Mon. Wea. Rev., 127, 1566-1585.

Zhao, M., I. M. Held, S.-J. Lin, and G. A. Vecchi, 2009: Simulations of global hurricane climatology, interannual variability, and response to global warming using a $50-\mathrm{km}$ resolution GCM. J. Climate, 22, 6653-6678.

Zhou, X., and B. Wang, 2009: From concentric eyewall to annular hurricane: A numerical study with the cloud-resolved WRF model Geophys. Res. Lett., 36, L03802, doi:10.1029/ 2008GL036854. 